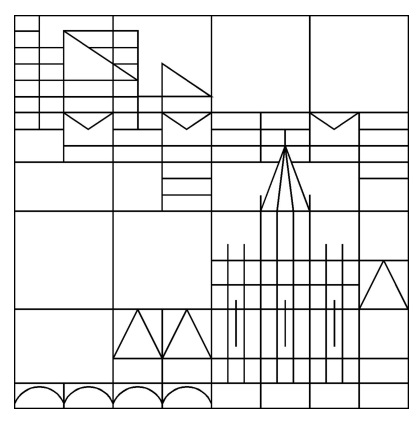

\title{
Parabolic boundary value problems connected with Newton's polygon and some problems of crystallization
}

\author{
Robert Denk \\ Leonid R. Volevich
}

Konstanzer Schriften in Mathematik und Informatik

Nr. 243, Februar 2008

ISSN 1430-3558

Konstanzer Online-Publikations-System (KOPS)

URL: http://www.ub.uni-konstanz.de/kops/volltexte/2008/4912/

URN: http://nbn-resolving.de/urn:nbn:de:bsz:352-opus-49127

\footnotetext{
(C) Fachbereich Mathematik und Statistik

(C) Fachbereich Informatik und Informationswissenschaft

Universität Konstanz

Fach D 188, 78457 Konstanz, Germany

E-Mail: preprints@informatik.uni-konstanz.de

WWW: http://www.informatik.uni-konstanz.de/Schriften/
} 



\title{
PARABOLIC BOUNDARY VALUE PROBLEMS CONNECTED WITH NEWTON'S POLYGON AND SOME PROBLEMS OF CRYSTALLIZATION
}

\author{
R. DENK, L. R. VOLEVICH ${ }^{1}$
}

\begin{abstract}
A new class of boundary value problems for parabolic operators is introduced which is based on the Newton polygon method. We show unique solvability and a priori estimates in corresponding $L_{2}$-Sobolev spaces. As an application, we discuss some linearized free boundary problems arising in crystallization theory which do not satisfy the classical parabolicity condition. It is shown that these belong to the new class of parabolic boundary value problems, and two-sided estimates for their solutions are obtained.
\end{abstract}

\section{INTRODUCTION}

This paper is motivated by several linearized boundary value problems from mathematical physics arising, e.g., in crystallization theory. In particular, we will consider the Stefan problem with Gibbs-Thomson correction and the Cahn-Hilliard equation with dynamics boundary conditions. These two problems have common specific features: the equation in the interior of the domain is of a rather simple structure and is parabolic in the sense of Petrovskii, but the boundary operators do not satisfy the condition of Shapiro-Lopatinskii type.

In fact, in these and similar examples there are several reasons why the ShapiroLopatinskii conditions cannot by satisfied:

- The boundary conditions are dynamic, i.e. time derivatives appear in the boundary conditions.

- In the case of a free boundary value problem, the free boundary is usually transformed by the Hanzawa transform to a fixed boundary. This leads to an additional unknown function on the boundary.

- The boundary operators have an inherent inhomogeneity. In the examples mentioned above, the equation in the interior of the domain is $2 b$-parabolic in the sense of Petrovskii, so the time derivative has weight $2 b$ with respect to space derivatives. In the boundary operators, however, the time derivative appears again but with a different weight $2 b^{\prime} \neq 2 b$.

The third reason is the most serious one. Due to the missing (quasi-)homogeneity of the corresponding symbols, uniform a priori estimates cannot be obtained by usual arguments.

The goal of this paper is to include these two problems in a rather general class of parabolic problems in which also "lower-order" terms in the boundary conditions play an important role.

Date: November 13, 2007.

1 The second author was supported by Russian Foundation of Basic Research, grant 06-0100096. 
The main idea of our approach is the following. As it is well known, even for scalar operators the theory of parabolic (as well as elliptic and parameterelliptic) problems is deeply connected with the theory of mixed order systems of pseudodifferential operators acting on the boundary. The matrix-symbol of this system is the so-called Lopatinskii matrix. In standard parabolic problems, this system is parabolic in the sense of Solonnikov [10]. We study a more general class of parabolic boundary value problems replacing systems parabolic in the sense of [10] by a more general class of N-parabolic systems which were studied by the second author in [12]. In the definition and analysis of these systems the notion of the Newton polygon plays a crucial role.

We now come to the formulation of the class of boundary value problems under consideration. To simplify the presentation, we will restrict ourselves to the model problem in half-space $\mathbb{R}_{+}^{n}:=\left\{x=\left(x^{\prime}, x_{n}\right) \in \mathbb{R}^{n}: x_{n}>0\right\}$ with boundary $\mathbb{R}^{n-1}$.

In the interior of the domain we will consider an equation of the form

$$
\begin{array}{rlrl}
A\left(D_{x}, D_{t}\right) u(x, t) & =f(x, t) & & \left(x \in \mathbb{R}_{+}^{n}, t \in \mathbb{R}\right), \\
u(x, t) & =f(x, t)=0 \quad & \left(x \in \mathbb{R}_{+}^{n}, t<0\right) .
\end{array}
$$

Here $D_{x}=-i\left(\frac{\partial}{\partial x_{1}}, \ldots, \frac{\partial}{\partial x_{n}}\right)$ and $D_{t}=-i \frac{\partial}{\partial t}$. We will assume that $A$ is $2 b$-parabolic in the sense of Petrovskii and we will denote the order of $A$ by $2 \mathrm{~m}$. On the boundary we have $\kappa$ additional functions $\sigma_{1}, \ldots, \sigma_{\kappa}$. Consequently, we need $m+\kappa$ boundary conditions

$$
\begin{array}{r}
B_{j}\left(D_{x}, D_{t}\right) u\left(x^{\prime}, t\right)+\sum_{k=1}^{\kappa} C_{j k}\left(D_{x^{\prime}}, D_{t}\right) \sigma_{k}\left(x^{\prime}, t\right)=g_{j}\left(x^{\prime}, t\right) \\
\left(j=1, \ldots, m+\kappa, x^{\prime} \in \mathbb{R}^{n-1}, t \in \mathbb{R}\right) .
\end{array}
$$

Again we assume $\sigma_{k}\left(x^{\prime}, t\right)=g_{j}\left(x^{\prime}, t\right)=0$ for $t<0$. The $2 b$-parabolicity condition on $A$ means that

$$
A_{0}(\xi, \tau) \neq 0 \quad\left((\xi, \tau) \in \mathbb{R}^{n} \times \mathbb{C} \text { with }|\xi|^{2 b}+|\tau|=1, \operatorname{Im} \tau \leq 0\right),
$$

where $A_{0}$ is the principal part of $A$. Here for the definition of the principal part we have to assign the weight $2 b$ to the co-variable $\tau$. For simplicity we will suppose that our operators have constant coefficients and have no lower terms, so that $A_{0}$ coincides with $A$.

The two main examples of boundary value problems of the form (1-1)-(1-2) are the Stefan problem and the Cahn-Hilliard equation. First, the linearized Stefan problem with Gibbs-Thomson correction is given by

$$
\begin{aligned}
\partial_{t} u(x, t)-\Delta u(x, t) & =f(x, t) \quad\left(t>0, x \in \mathbb{R}_{+}^{n}\right), \\
u\left(x^{\prime}, 0, t\right)+\Delta^{\prime} \sigma\left(x^{\prime}, t\right) & =g\left(x^{\prime}, t\right) \quad\left(t>0, x^{\prime} \in \mathbb{R}^{n-1}\right), \\
\partial_{n} u\left(x^{\prime}, 0, t\right)-\partial_{t} \sigma\left(x^{\prime}, t\right) & =h\left(x^{\prime}, t\right) \quad\left(t>0, x^{\prime} \in \mathbb{R}^{n-1}\right), \\
u(x, 0) & =u_{0} \quad\left(x \in \mathbb{R}^{n}\right), \\
\sigma\left(x^{\prime}, 0\right) & =\sigma_{0} \quad\left(x^{\prime} \in \mathbb{R}^{n-1}\right) .
\end{aligned}
$$

Here $\Delta^{\prime}$ is the Laplace operator on the boundary $\mathbb{R}^{n-1}$ and $\partial_{n}$ stands for the normal derivative. 
In the case of zero initial conditions we can consider the problem on the whole time axis, supposing that

$$
\begin{aligned}
u(x, t)=f(x, t)=0 & \left(x \in \mathbb{R}_{+}^{n}, t<0\right), \\
\sigma\left(x^{\prime}, t\right)=g\left(x^{\prime}, t\right)=h\left(x^{\prime}, t\right)=0 & \left(x^{\prime} \in \mathbb{R}^{n-1}, t<0\right) .
\end{aligned}
$$

The Stefan problem (1-4) was studied in a number of papers. Recently it was treated in detail by Escher-Prüss-Simonett in [7]. It was shown that in appropriate solution spaces this equation is uniquely solvable. Whereas in [7] the approach is semigroup based, we will see below that we can understand the structure of this boundary value problem and of the solution spaces in terms of the Newton polygon. Below we will show that the classical parabolicity condition is not satisfied for the Stefan problem (1-4).

Now let us come to the linearized Cahn-Hilliard equation with dynamic boundary conditions. It is given by

$$
\begin{array}{rlrl}
\partial_{t} u(x, t)+\Delta^{2} u(x, t) & =f(x, t) & & \left(t>0, x \in \mathbb{R}_{+}^{n}\right), \\
\partial_{n} \Delta u\left(x^{\prime}, 0, t\right) & =g\left(x^{\prime}, t\right) & & \left(t>0, x^{\prime} \in \mathbb{R}^{n-1}\right), \\
\partial_{t} u\left(x^{\prime}, 0, t\right)+\partial_{n} u\left(x^{\prime}, 0, t\right)-\Delta^{\prime} u\left(x^{\prime}, 0, t\right) & =h\left(x^{\prime}, t\right) & \left(t>0, x^{\prime} \in \mathbb{R}^{n-1}\right), \\
u(x, 0) & =u_{0} \quad\left(t=0, x \in \mathbb{R}_{+}^{n}\right) .
\end{array}
$$

The solvability of this problem in appropriate Sobolev spaces was investigated by Prüss-Racke-Zheng in [8]. Again the method was based on a semigroup approach. We will see below that also the Cahn-Hilliard equation (1-5) fits into the context of parabolic problems connected with the Newton polygon.

The paper is organized in the following way. In Section 2, we will discuss the definition of standard $2 b$-parabolic problems and the Lopatinskii matrix. In particular, we will show that the linearized problems of crystallization are not $2 b$-parabolic. In Section 3, the notion of N-parabolic problems will be defined which is connected with the Newton polygon. We will see that both examples belong to this larger class of parabolic boundary value problems. The last three sections of the paper are devoted to solvability results. After defining the Sobolev spaces connected with (1-4)-(1-5) in Section 4, we will prove a general result on unique solvability and a priori estimates in Section 5. The application to the two examples can be found in Section 6.

In subsequent publications we plan to treat the problems with variable coefficients using the construction of an exact parametrix of the Dirichlet problem for parabolic operators. In the case of problems (1-4) and (1-5) estimates in scales of Besov spaces will be given.

Below we will denote the co-variable to $t$ by $\tau$ and the co-variables to $x \in \mathbb{R}^{n}$ and $x^{\prime} \in \mathbb{R}^{n-1}$ by $\xi \in \mathbb{R}^{n}$ and $\xi^{\prime}:=\left(\xi_{1}, \ldots, \xi_{n-1}\right)$. In the following, $C$ stands for an unspecified constant which may vary from one appearance to the other but which is independent of the free variables. The notion $f \approx g$ means that there exists a positive constant $C$ for which $C^{-1} f \leq g \leq C f$.

\section{2. $2 b$-PARABOLIC PROBLEMS. The LOPATINSKII MATRIX}

In this section we introduce the main object of the further investigation - the Lopatinskii matrix of the boundary value problem (1-1)-(1-2). We define standard 
$2 b-$ parabolic problems and show that the problems (1-4) and (1-5) do not belong to this class.

2.1. Remarks about the factorization. We assume that the operator $A\left(D_{x}, D_{\tau}\right)$ is $2 b$-parabolic. We will need the factorization of the symbol $A(\xi, \tau)$, i, e. its representation in the form

$$
A\left(\xi^{\prime}, \xi_{n}, \tau\right)=A^{+}\left(\xi^{\prime}, \xi_{n}, \tau\right) A^{-}\left(\xi^{\prime}, \xi_{n}, \tau\right)
$$

with

$$
A^{+}\left(\xi^{\prime}, \xi_{n}, \tau\right):=\prod_{j=1}^{m}\left(z-z_{j}^{+}\left(\xi^{\prime}, \tau\right)\right)=z^{m}+\sum_{\ell=1}^{m} a_{\ell}\left(\xi^{\prime}, \tau\right) z^{m-\ell} .
$$

Here $z_{j}^{+}\left(\xi^{\prime}, \tau\right)$ are the roots of $A\left(\xi^{\prime}, \cdot, \tau\right)$ with positive imaginary part.

In the following, the (quasi-)homogeneities of the symbols will be important, so we want to define $\rho$-homogeneity. For a weight $\rho>0$, a function $F: \mathbb{R}^{n} \times \mathbb{C} \rightarrow \mathbb{C}$ will be called $\rho$-homogeneous of degree $d \in \mathbb{R}$ if

$$
F\left(\eta \xi, \eta^{\rho} \tau\right)=\eta^{d} F(\xi, \tau) \quad\left(\xi \in \mathbb{R}^{n} \backslash\{0\}, \tau \in \mathbb{C} \backslash\{0\}, \eta>0\right) .
$$

In this case we will call $d$ the $\rho$-order of the symbol $F$ and write $\operatorname{ord}_{\rho} F(\xi, \tau):=d$. Here $\rho$ denotes the weight of the co-variable $\tau$ when $\xi$ has weight 1 . If $F$ is a sum of quasi-homogeneous terms the $\rho$-order is defined in an obvious way where again $\tau$ has weight $\rho$.

As the symbol $A\left(\xi^{\prime}, z, \tau\right)$ is $2 b$-homogeneous in $\left(\xi^{\prime}, z, \tau\right)$, the same is true for its roots. Therefore, we have

$$
\begin{aligned}
\left|z_{j}^{+}\left(\xi^{\prime}, \tau\right)\right| & \leq C\left(|\tau|^{\frac{1}{2 b}}+\left|\xi^{\prime}\right|\right), \\
\operatorname{Im} z_{j}^{+}\left(\xi^{\prime}, \tau\right) & \geq C\left(|\tau|^{\frac{1}{2 b}}+\left|\xi^{\prime}\right|\right)
\end{aligned}
$$

for $j=1, \ldots, m$. We can represent $z_{j}^{+}$in the form

$$
z_{j}^{+}\left(\xi^{\prime}, \tau\right)=\left(|\tau|^{\frac{1}{2 b}}+\left|\xi^{\prime}\right|\right) \tilde{z}_{j}^{+}\left(\xi^{\prime}, \tau\right)
$$

where $\tilde{z}_{j}^{+}$is a $2 b$-homogeneous function of $\left(\xi^{\prime}, \tau\right)$ of degree zero.

From this we obtain the analogous representation of the coefficients $a_{\ell}\left(\xi^{\prime}, \tau\right)$ :

$$
a_{\ell}\left(\xi^{\prime}, \tau\right)=\left(|\tau|^{\frac{1}{2 b}}+\left|\xi^{\prime}\right|\right)^{\ell} \tilde{a}_{\ell}\left(\xi^{\prime}, \tau\right)
$$

where $\tilde{a}_{\ell}$ is a $2 b$-homogeneous function of $\left(\xi^{\prime}, \tau\right)$ of degree 0 . We will not suppose, in principle, that the polynomials $B_{j}(\xi, \tau)$ and $C_{j k}\left(\xi^{\prime}, \tau\right)$ are quasi-homogeneous.

2.2. The Lopatinskii matrix of the problem (1-1)-(1-2). As in the classical (quasi-homogeneous) situation, the Lopatinskii matrix of (1-1)-(1-2) is constructed using the remainders of the symbols of the boundary operators modulo $A^{+}\left(\xi^{\prime}, z, \tau\right)$. For $\ell \geq m$ we have

$$
z^{\ell} \equiv \sum_{k=1}^{m} \gamma_{\ell k}\left(\xi^{\prime}, \tau\right) z^{k-1} \bmod A^{+}\left(\xi^{\prime}, z, \tau\right) .
$$

From the rule of division of polynomials it follows that the coefficients $\gamma_{\ell k}\left(\xi^{\prime}, \tau\right)$ are $2 b$-homogeneous polynomials of $z_{1}^{+}, \ldots, z_{m}^{+}$of degree $\ell+1-k$ and can be represented in the form

$$
\gamma_{\ell k}\left(\xi^{\prime}, \tau\right)=\left(|\tau|^{\frac{1}{2 b}}+\left|\xi^{\prime}\right|\right)^{\ell+1-k} \tilde{\gamma}_{\ell k}\left(\tau, \xi^{\prime}\right),
$$


where $\tilde{\gamma}_{\ell k}$ are $2 b$-homogeneous of degree 0 . Further we replace in the symbols $B_{j}\left(\xi^{\prime}, z, \tau\right)$ every power $z^{\ell}$ with $\ell \geq m$ by their remainder modulo $A^{+}\left(\xi^{\prime}, z, \tau\right)$. We get

$$
B_{j}\left(\xi^{\prime}, z, \tau\right) \equiv \tilde{B}_{j}\left(\xi^{\prime}, z, \tau\right) \bmod A^{+}\left(\xi^{\prime}, z, \tau\right)
$$

with

$$
\tilde{B}_{j}\left(\xi^{\prime}, z, \tau\right)=\sum_{k=1}^{m} b_{j k}\left(\xi^{\prime}, \tau\right) z^{k-1} .
$$

From the construction we see that the functions $b_{j k}\left(\xi^{\prime}, \tau\right)$ are polynomials in $\xi^{\prime}, \tau$ and in the coefficients $\gamma_{\ell k}\left(\xi^{\prime}, \tau\right)$ which themselves are polynomials in $|\tau|^{\frac{1}{2 b}}$ and $\left|\xi^{\prime}\right|$ with coefficients depending on $\tau\left(|\tau|^{1 / 2 b}+\left|\xi^{\prime}\right|\right)^{-2 b}$ and $\xi^{\prime}\left(|\tau|^{1 / 2 b}+\left|\xi^{\prime}\right|\right)^{-1}$. Therefore, we can define the $2 b$-order of $b_{j k}$. We have

$$
\operatorname{ord}_{2 b} b_{j k} \leq m_{j}+1-k \quad(j=1, \ldots, m+\kappa, k=1, \ldots, m) .
$$

Here we have set $m_{j}:=\operatorname{ord}_{2 b} B_{j}(\xi, \tau)$.

The matrix-function

$$
L\left(\xi^{\prime}, \tau\right)=\left(L_{j k}\left(\xi^{\prime}, \tau\right)\right)_{j, k=1, \ldots, m+\kappa}
$$

with

$$
L_{j k}\left(\xi^{\prime}, \tau\right):= \begin{cases}b_{j k}\left(\xi^{\prime}, \tau\right) & (j=1, \ldots, m+\kappa, k=1, \ldots, m), \\ C_{j, k-m}\left(\xi^{\prime}, \tau\right) & (j=1, \ldots, m+\kappa, k=m+1, \ldots, m+\kappa)\end{cases}
$$

is called the Lopatinskii matrix of the problem (1-1)-(1-2).

The elements of the Lopatinskii matrix are holomorphic functions in $\tau$. We will prove this statement at the end of this section.

2.3. The $2 b-$ parabolicity condition for the boundary value problem (1-1)(1-2). As it was mentioned above, although the elements of the Lopatinskii matrix are algebraic functions, their orders. Then we can define the $2 b$-principal part $L^{0}\left(\xi^{\prime}, \tau\right)$ of $L\left(\xi^{\prime}, \tau\right)$. If we pose

$$
s_{j}:=m_{j}+1-m \quad(j=1, \ldots, m+\kappa), \quad t_{k}:=m-k \quad(k=1, \ldots, m)
$$

we have $\operatorname{ord}_{2 b} b_{j k} \leq s_{j}+t_{k}$. For the operators $C_{j k}$ we set

$$
t_{k}:=\max \left\{\operatorname{ord}_{2 b} C_{j, k-m}-s_{j}: j=1, \ldots, m+\kappa\right\} \quad(k=m+1, \ldots, m+\kappa) .
$$

In this way we obtain

$$
\text { ord } L_{j k}\left(\xi^{\prime}, \tau\right) \leq s_{j}+t_{k} \quad(j, k=1, \ldots, m+\kappa) .
$$

Let $\pi_{2 b} L_{j k}\left(\xi^{\prime}, \tau\right)$ be the principal part of $L_{j k}\left(\xi^{\prime}, \tau\right)$ with the weight of $\tau$ being $2 b$. Then the principal part of the Lopatinskii matrix is defined as $L^{0}\left(\xi^{\prime}, \tau\right)=$ $\left(L_{j k}^{0}\left(\xi^{\prime}, \tau\right)\right)_{j, k=1, \ldots, m+\kappa}$ with

$$
L_{j k}^{0}\left(\xi^{\prime}, \tau\right):= \begin{cases}\pi_{2 b} L_{j k}\left(\xi^{\prime}, \tau\right) & \text { if } \operatorname{ord}_{2 b} L_{j k}=s_{j}+t_{k}, \\ 0 & \text { if } \operatorname{ord}_{2 b} L_{j k}<s_{j}+t_{k} .\end{cases}
$$

Definition 2.1. We say that problem (1-1)-(1-2) satisfies the $2 b-$ parabolicity condition if the following conditions hold.

(i) $A\left(D_{x}, D_{t}\right)$ is $2 b$-parabolic. 
(ii) $\operatorname{det} L^{0}\left(\xi^{\prime}, \tau\right)=\pi_{2 b} \operatorname{det} L\left(\xi^{\prime}, \tau\right)$, i.e. we have

$$
\operatorname{ord}_{2 b} \operatorname{det} L\left(\xi^{\prime}, \tau\right)=\sum_{j=1}^{m+\kappa}\left(s_{j}+t_{j}\right) .
$$

(iii) For all $\xi^{\prime} \in \mathbb{R}^{n-1}$ and $\tau \in \mathbb{C}$ with $|\tau|+\left|\xi^{\prime}\right|>0$ and $\operatorname{Im} \tau \leq 0$ we have

$$
\operatorname{det} L^{0}\left(\xi^{\prime}, \tau\right) \neq 0 \text {. }
$$

Conditions (ii),(iii) mean that the matrix $L\left(\xi^{\prime}, \tau\right)$ is parabolic in the sense of Solonnikov [10].

Remark 2.2. For parabolic problems the analog of the Shapiro-Lopatinskii condition from elliptic theory was introduced in the papers of Eidelman (see [4]) and Solonnikov (see [10]). Agranovich and Vishik [3] studied elliptic problems with parameter and introduced the parameter-ellipticity condition which formally coincides with Eidelman-Solonnikov condition. The parameter-ellipticity condition was independently introduced by Agmon [1]. In the context of problems with parameter we shall call it Agmon-Agranovich-Vishik (AAV) condition.

2.4. The problems (1-4) and (1-5) are not 2b-parabolic. We will see now that neither the Stefan problem with Gibbs-Thomson correction nor the Cahn-Hilliard equation with dynamic boundary condition satisfy condition (iii) of Definition 2.1.

Let us start with the Stefan problem (1-4). Using the factorization of the symbol of the heat operator

$$
i \tau+\left|\xi^{\prime}\right|^{2}+\xi_{n}^{2}=\left(\xi_{n}-i \sqrt{\left|\xi^{\prime}\right|^{2}+i \tau}\right)\left(\xi_{n}+i \sqrt{\left|\xi^{\prime}\right|^{2}+i \tau}\right),
$$

we calculate the Lopatinskii matrix

$$
L\left(\tau, \xi^{\prime}\right)=\left(\begin{array}{cc}
1 & -\left|\xi^{\prime}\right|^{2} \\
-\sqrt{\left|\xi^{\prime}\right|^{2}+i \tau} & -i \tau
\end{array}\right) .
$$

We can take $s_{1}=0, s_{2}=1, t_{1}=0, t_{2}=2$, and the principal part of $L\left(\tau, \xi^{\prime}\right)$ is given by

$$
L^{0}\left(\tau, \xi^{\prime}\right)=\left(\begin{array}{cc}
1 & -\left|\xi^{\prime}\right|^{2} \\
-\sqrt{\left|\xi^{\prime}\right|^{2}+i \tau} & 0
\end{array}\right) .
$$

Condition (ii) of Definition 2.1 is satisfied, but condition (iii) is violated because

$$
\operatorname{det} L^{0}\left(\tau, \xi^{\prime}\right)=-\left|\xi^{\prime}\right|^{2} \sqrt{\left|\xi^{\prime}\right|^{2}+i \tau}=0 \text { for }\left|\xi^{\prime}\right|=0,|\tau|>0 .
$$

Now let us consider the Cahn-Hilliard problem (1-5). We factorize

$$
i \tau+\left(\left|\xi^{\prime}\right|^{2}+\xi_{n}^{2}\right)^{2}=A^{+}\left(\xi^{\prime}, \xi_{n}, \tau\right) \cdot A^{-}\left(\xi^{\prime}, \xi_{n}, \tau\right)
$$

with

$$
A^{+}\left(\xi^{\prime}, \xi_{n}, \tau\right)=\left(\xi_{n}-z_{1}\left(\xi^{\prime}, \tau\right)\right) \cdot\left(\xi_{n}-z_{2}\left(\xi^{\prime}, \tau\right)\right) .
$$

The Lopatinskii matrix is given by

$$
L\left(\xi^{\prime}, \tau\right)=\left(\begin{array}{cc}
i z_{1} z_{2}\left(z_{1}+z_{2}\right) & -i\left(z_{1}^{2}+z_{2}^{2}+z_{1} z_{2}+\left|\xi^{\prime}\right|^{2}\right) \\
i \tau+\left|\xi^{\prime}\right|^{2} & i
\end{array}\right) .
$$

In this case $\tau$ has weight 4 and $m_{1}=3, m_{2}=4$. Thus $s_{1}=2, s_{2}=3, t_{1}=1, t_{2}=0$, and the principal part of $L$ is equal to

$$
L^{0}\left(\xi^{\prime}, \tau\right)=\left(\begin{array}{cc}
i z_{1} z_{2}\left(z_{1}+z_{2}\right) & -i\left(z_{1}^{2}+z_{2}^{2}+z_{1} z_{2}+\left|\xi^{\prime}\right|^{2}\right) \\
i \tau & 0
\end{array}\right) .
$$


Obviously, we have $\operatorname{det} L^{0}\left(\xi^{\prime}, \tau\right)=0$ for $\tau=0$ and arbitrary $\xi^{\prime}$. Again the $2 b$ parabolicity condition is not satisfied.

2.5. The Lopatinskii matrix is holomorphic in $\tau$. According to the definition, the elements of the Lopatinskii matrix $L\left(\xi^{\prime}, \tau\right)$ are remainders after division of polynomials by $A^{+}\left(z, \xi^{\prime}, \tau\right)$ defined in (2-1). From the elementary algorithm of division with remainder easily follows that we only need to check that the coefficients $a_{\ell}\left(\xi^{\prime}, \tau\right)$ appearing in (2-1) are holomorphic in $\tau$.

According to the classical Vieta formula the coefficients

$$
a_{\ell}\left(\xi^{\prime}, \tau\right)=(-1)^{\ell} \sum_{i_{1}, \ldots, i_{m-\ell}} z_{i_{1}}^{+}\left(\xi^{\prime}, \tau\right) \ldots z_{i_{m-\ell}}^{+}\left(\xi^{\prime}, \tau\right), \quad \ell=1, \ldots, m
$$

are symmetric functions of the roots $z_{1}^{+}, \ldots, z_{m}^{+}$and can be expressed by means of

$$
\sum_{j=1}^{m}\left(z_{j}^{+}\left(\xi^{\prime}, \tau\right)\right)^{r}, \quad r=1, \ldots, m-1 .
$$

To prove that sums (2-4) are holomorphic in $\tau$ we use the standard argument of the Weierstrass preparation theorem. We choose a contour $\gamma_{+}$in the half-plane $\operatorname{Im} z>0$ enveloping all the roots $z_{1}^{+}, \ldots, z_{m}^{+}$. Then

$$
\sum_{j=1}^{m}\left(z_{j}^{+}\left(\xi^{\prime}, \tau\right)\right)^{r}=\frac{1}{2 \pi i} \int_{\gamma_{+}} \frac{z^{r} \partial_{z} A\left(\xi^{\prime}, z, \tau\right)}{A\left(\xi^{\prime}, z, \tau\right)} d z .
$$

The expression under the sign of the integral is a rational function of $\tau$ and has no poles for $\operatorname{Im} \tau<0$ and $z \in \gamma_{+}$. From this follows that the left-hand side of (2-5) is holomorphic in $\tau$ and, consequently, the same holds for the elements of the Lopatinskii matrix.

\section{N-PARABOLIC BOUNDARY VALUE PROBLEMS}

In this section we introduce a more general class of parabolic problems such that the examples above belong to it. The main idea is to replace the traditional principal part of the Lopatinskii matrix by a principal part connected with the Newton polygon of $\operatorname{det} L$. We shall need some definitions and results from [5] and [12].

3.1. Newton's polygon and N-parabolic polynomials. Consider a polynomial

$$
P(\xi, \tau)=\sum_{\alpha, j} p_{\alpha j} \xi^{\alpha} \tau^{j}
$$

in the variables $\xi \in \mathbb{R}^{n}$ and $\tau \in \mathbb{C}$. Then the Newton polygon $N(P)$ of the polynomial $P$ is defined as the convex hull of all points $(|\alpha|, j)$ for which $p_{\alpha j} \neq 0$, the projections of all these points to the coordinate axes and the origin. The following definition is taken from [5].

Definition 3.1. The polynomial $P(\xi, \tau)$ is called N-parabolic if the following conditions hold.

(i) The Newton polygon $N(P)$ has no edges parallel to the coordinate axes (except the trivial ones). 
(ii) There exists a $\tau_{0}<0$ such that the estimate

$$
|P(\xi, \tau)| \geq C \sum_{(i, j) \in N(P) \cap \mathbb{Z}^{2}}|\xi|^{i}|\tau|^{j} \quad\left(\xi \in \mathbb{R}^{n}, \operatorname{Im} \tau \leq \tau_{0}\right)
$$

holds. Here the sum runs over all integer points belonging to the Newton polygon.

3.2. N-parabolic polynomial matrices. N-parabolic polynomials can be included in the class of so-called N-parabolic matrices (see [12]). For this, we consider a polynomial matrix

$$
P(\xi, \tau)=\left(P_{j k}(\xi, \tau)\right)_{j, k=1, \ldots, N}
$$

and write the determinant of $P$ in the form

$$
\operatorname{det} P(\xi, \tau)=\sum_{\gamma} \pm P_{1, \gamma(1)} \ldots P_{N, \gamma(N)},
$$

where $\gamma$ runs through all permutations of the set $\{1,2, \ldots, N\}$. Assigning weight $\rho$ to the variable $\tau$ and weight 1 to the variables $\xi$ we define

$$
R(\rho):=\max _{\gamma}\left(\operatorname{ord}_{\rho} P_{1, \gamma(1)}+\cdots+\operatorname{ord}_{\rho} P_{N, \gamma(N)}\right) .
$$

Definition 3.2. a) The matrix $P(\xi, \tau)$ is called totally non-degenerate if for every $\rho>0$ we have

$$
R(\rho)=\operatorname{ord}_{\rho} \operatorname{det} P(\xi, \tau) .
$$

b) The matrix $P(\xi, \tau)$ is called N-parabolic if the following conditions hold.

(i) $P(\xi, \tau)$ is totally non-degenerate.

(ii) The determinant $\operatorname{det} P(\xi, \tau)$ is N-parabolic.

For N-parabolic matrices $P(\xi, \tau)$ it is possible to define the principal part $P_{\rho}(\xi, \tau)$ for every fixed weight $\rho>0$. As it was noted in [12], according to [11] for every $\rho$ there exist real numbers $s_{j}(\rho)$ and $t_{k}(\rho)(j, k=1, \ldots, N)$ for which

$$
\begin{aligned}
\operatorname{ord}_{\rho} P_{j k}(\xi, \tau) & \leq s_{j}(\rho)+t_{k}(\rho) \quad(j, k=1, \ldots, N), \\
\quad \operatorname{ord}_{\rho} \operatorname{det} P & =\sum_{j=1}^{N}\left(s_{j}(\rho)+t_{j}(\rho)\right) .
\end{aligned}
$$

The principal part $P_{\rho}(\xi, \tau)$ is defined in the standard way.

The matrix $P(\xi, \tau)$ is called positively totally non-degenerate if the above functions $s_{i}(\rho), t_{i}(\rho), i=1, \ldots, N$, can be chosen nonnegative. Replacing in Definition $3.2 \mathrm{~b}$ ) totally non-degenerate matrices by positively totally non-degenerate, we define positively N-parabolic matrices.

3.3. N-parabolic boundary value problems. We return to problem (1-1)-(1-2). Formally the above definitions cannot be applied to the Lopatinskii matrix of this problem, because its elements are not polynomials but, in principal, algebraic functions. In fact, Definition 3.1 and Definition 3.2 use only the possibility to calculate $\operatorname{ord}_{\rho} P_{i j}$ for each element $P_{i j}$ and each $\rho>0$.

The special structure of the elements of the Lopatinskii matrix permits to calculate these orders. Indeed, as it was mentioned above, the elements of this matrix are polynomials in $\left(\xi^{\prime}, \tau\right)$ and the roots $z_{1}^{+}, \ldots, z_{m}^{+}$. Setting $\operatorname{ord}_{\rho} z_{j}^{+}=\rho / 2 b$ for $\rho \geq 2 b$ and $\operatorname{ord}_{\rho} z_{j}^{+}=1$ for $\rho \leq 2 b$ we define $\operatorname{ord}_{\rho}$ for each element of the Lopatinskii matrix. So we can give 
Definition 3.3. The problem (1-1)-(1-2) is called N-parabolic if the following conditions hold.

(i) $A\left(D_{x}, D_{t}\right)$ is $2 b$-parabolic.

(ii) The Lopatinskii matrix $L\left(\xi^{\prime}, \tau\right)$ is N-parabolic in the sense of Definition 3.2.

3.4. Reduction of an N-parabolic boundary value problem to an N-parabolic system on the boundary. For simplicity we start from problem (1-1)-(1-2) with $f \equiv 0$ and without additional functions at the boundary conditions,

$$
\begin{aligned}
A\left(D_{x}, D_{t}\right) u(x, t) & =0 & & \left(x \in \mathbb{R}_{+}^{n}, t \in \mathbb{R}\right), \\
u(x, t) & =0 & & \left(x \in \mathbb{R}_{+}^{n}, t<0\right), \\
B_{j}\left(D_{x}, D_{t}\right) u\left(x^{\prime}, t\right) & =g_{j}\left(x^{\prime}, t\right) & & \left(j=1, \ldots, m, x^{\prime} \in \mathbb{R}^{n-1}, t \in \mathbb{R}\right) .
\end{aligned}
$$

Here $A\left(D_{x}, D_{t}\right)$ is, as above, a $2 b$-parabolic operator with constant coefficients of order $2 m$, and $B_{j}\left(D_{x}, D_{t}\right)$ are operators with constant coefficients. We do not assume that the boundary operators are quasi-homogeneous.

Let $h_{j}\left(x^{\prime}, t\right), j=1, \ldots, m$, be functions with $h_{1}\left(x^{\prime}, t\right)=\cdots=h_{m}\left(x^{\prime}, t\right)=0$ for $t<0$. Then we denote by $U\left(h_{1}, \ldots, h_{m}, x, t\right)$ the solution of the Dirichlet problem

$$
\begin{aligned}
A\left(D_{x}, D_{t}\right) U\left(h_{1}, \ldots, h_{m}, x, t\right) & =0 \quad\left(x \in \mathbb{R}_{+}^{n}, t \in \mathbb{R}\right), \\
U\left(h_{1}, \ldots, h_{m}, x, t\right) & =0 \quad\left(x \in \mathbb{R}_{+}^{n}, t<0\right), \\
D_{n}^{j-1} U\left(h_{1}, \ldots, h_{m}, x^{\prime}, t\right) & =h_{j}\left(x^{\prime}, t\right) \quad\left(j=1, \ldots, m, x^{\prime} \in \mathbb{R}^{n-1}, t \in \mathbb{R}\right) .
\end{aligned}
$$

We shall seek the solution of (3-1)-(3-2) in the form $u(x, t)=U\left(h_{1}, \ldots, h_{m}, x, t\right)$ with functions $h_{1}, \ldots, h_{m}$ to be found.

Lemma 3.4. For $j=1, \ldots, m$ we have

$$
\left.B_{j}\left(D_{x}, D_{t}\right) U\left(h_{1}, \ldots, h_{m}, x, t\right)\right|_{x_{n}=0}=\sum_{k=1}^{m} L_{j k}\left(D_{x^{\prime}}, D_{t}\right) h_{k}\left(x^{\prime}, t\right)
$$

hold.

Proof. Starting from the relation

$$
A^{+}\left(\xi^{\prime}, z, \tau\right)=z^{m}+\sum_{\ell=1}^{m} a_{\ell}\left(\xi^{\prime}, \tau\right) z^{m-\ell}=: \sum_{\ell=0}^{m} a_{\ell}\left(\xi^{\prime}, \tau\right) z^{m-\ell},
$$

we define

$$
M_{k}\left(\xi^{\prime}, z, \tau\right):=\sum_{\ell=0}^{k} a_{\ell}\left(\xi^{\prime}, \tau\right) z^{k-\ell} \quad(k=0, \ldots, m-1) .
$$

As it was noted in [2] (see Chapter 1, Section 1),

$$
\frac{1}{2 \pi i} \int_{\gamma_{+}} \frac{z^{\ell-1} M_{m-k}\left(\xi^{\prime}, z, \tau\right)}{A^{+}\left(\xi^{\prime}, z, \tau\right)} d z=\delta_{k \ell}, \quad(k, \ell=1, \ldots, m),
$$

where the contour $\gamma_{+}$belongs to $\mathbb{C}_{+}$and contains all the zeros of $A^{+}\left(\xi^{\prime}, \cdot, \tau\right)$. We define the symbol

$$
G_{k}\left(\xi^{\prime}, \tau, x_{n}\right):=\frac{1}{2 \pi i} \int_{\gamma_{+}} \frac{M_{m-k}\left(\xi^{\prime}, z, \tau\right)}{A^{+}\left(\xi^{\prime}, z, \tau\right)} e^{i z x_{n}} d z \quad(k=1, \ldots, m)
$$


According to (3-6)

$$
U\left(h_{1}, \ldots, h_{m}, x, t\right)=\sum_{k=1}^{m} G_{k}\left(D_{x^{\prime}}, D_{t}, x_{n}\right) h_{k}\left(x^{\prime}, t\right),
$$

here $G_{k}\left(D_{x^{\prime}}, D_{t}, x_{n}\right)$ is the pseudodifferential operator in the variables $\left(x^{\prime}, t\right)$ with symbol (3-7) depending on the parameter $x_{n}$.

Now the left-hand side of (3-5) will be equal to

$$
\sum_{k=1}^{m}\left(\left.B_{j}\left(D_{x}, D_{t}\right) G_{k}\left(D_{x^{\prime}}, D_{t}, x_{n}\right)\right|_{x_{n}=0} h_{k}\left(x^{\prime}, t\right)\right) .
$$

Further

$$
\left.\sum_{k=1}^{m} B_{j}\left(D_{x}, D_{t}\right) G_{k}\left(D_{x^{\prime}}, D_{t}, x_{n}\right)\right|_{x_{n}=0}=\frac{1}{2 \pi i} \int_{\gamma_{+}} \frac{B_{j}\left(\xi^{\prime}, z, \tau\right) M_{m-k}\left(\xi^{\prime}, z, \tau\right)}{A^{+}\left(\xi^{\prime}, z, \tau\right)} d z .
$$

The integral on the right-hand side does not change if we replace $B_{j}\left(\xi^{\prime}, z, \tau\right)$ by $\tilde{B}_{j}\left(\xi^{\prime}, z, \tau\right)=\sum_{\ell=1}^{m} b_{j \ell}\left(\xi^{\prime}, \tau\right) z^{\ell-1}$. Now we obtain for $j, k=1, \ldots, m$

$$
\sum_{\ell=1}^{m} b_{j \ell}\left(\xi^{\prime}, \tau\right) \frac{1}{2 \pi i} \int_{\gamma_{+}} \frac{z^{\ell-1} M_{m-k}\left(\xi^{\prime}, z, \tau\right)}{A^{+}\left(\xi^{\prime}, z, \tau\right)} d z=\sum_{\ell=1}^{m} b_{j \ell}\left(\xi^{\prime}, \tau\right) \delta_{\ell k}=b_{j k}\left(\xi^{\prime}, \tau\right) .
$$

From the lemma we see that problem (3-1)-(3-2) is reduced to the system of pseudodifferential equations

$$
L\left(D_{x^{\prime}}, D_{t},\right)\left(h_{1}, \ldots, h_{m}\right)^{T}=\left(g_{1}, \ldots, g_{m}\right)^{T} .
$$

The approach described above can trivially be extended to the case of boundary conditions containing additional functions on the boundary of the form

$$
B_{j}\left(D_{x}, D_{t}\right) u\left(x^{\prime}, t\right)+\sum_{k=1}^{\kappa} C_{j k}\left(D_{x^{\prime}}, D_{t}\right) \sigma\left(x^{\prime}, t\right)=g_{j}\left(x^{\prime}, t\right) \quad(j=1, \ldots, m+\kappa) .
$$

Substituting in the boundary conditions the function $U\left(h_{1}, \ldots, h_{m}, x, t\right)$ and using the lemma we obtain the following system for $h_{1}, \ldots, h_{m}, \sigma_{1}, \ldots, \sigma_{\kappa}$.

$$
\sum_{k=1}^{m} L_{j k}\left(D_{x^{\prime}}, D_{t}\right) h_{k}+\sum_{k=1}^{\kappa} C_{j k}\left(D_{x^{\prime}}, D_{t}\right) \sigma_{k}=g_{j}, \quad(j=1, \ldots, m+\kappa) .
$$

3.5. N-parabolicity of the Stefan problem with Gibbs-Thomson correction. For the Stefan problem (1-4) the Lopatinskii matrix is given by (2-2), and its determinant equals to

$$
\left.\operatorname{det} L\left(\xi^{\prime}, \tau\right)=-\left(|\xi|^{2} \sqrt{\left|\xi^{\prime}\right|^{2}+i \tau}+i \tau\right)\right) .
$$

Note that for $\operatorname{Im} \tau \leq 0$ we have $\left|\sqrt{\left|\xi^{\prime}\right|^{2}+i \tau}\right| \approx\left|\xi^{\prime}\right|+|\tau|^{1 / 2}$. Therefore, the Newton polygon of $\operatorname{det} L\left(\xi^{\prime}, \tau\right)$ has the vertices $(0,0),(0,1),\left(2, \frac{1}{2}\right)$ and $(3,0)$ (see Figure 1).

For every $\rho>0$, we define $s_{j}(\rho)$ and $t_{k}(\rho)(j, k=1,2)$ by $s_{1}(\rho):=0, t_{1}(\rho):=0$ and

Then

$$
s_{2}(\rho):=\left\{\begin{array}{ll}
\frac{\rho}{2}, & \rho \geq 2, \\
1, & \rho \leq 2,
\end{array} \quad t_{2}(\rho):= \begin{cases}\frac{\rho}{2}, & \rho \geq 4, \\
2, & \rho \leq 4 .\end{cases}\right.
$$

$$
\operatorname{ord}_{\rho} L_{j k}\left(\tau, \xi^{\prime}\right) \leq s_{j}(\rho)+t_{k}(\rho) \quad(j, k=1,2)
$$




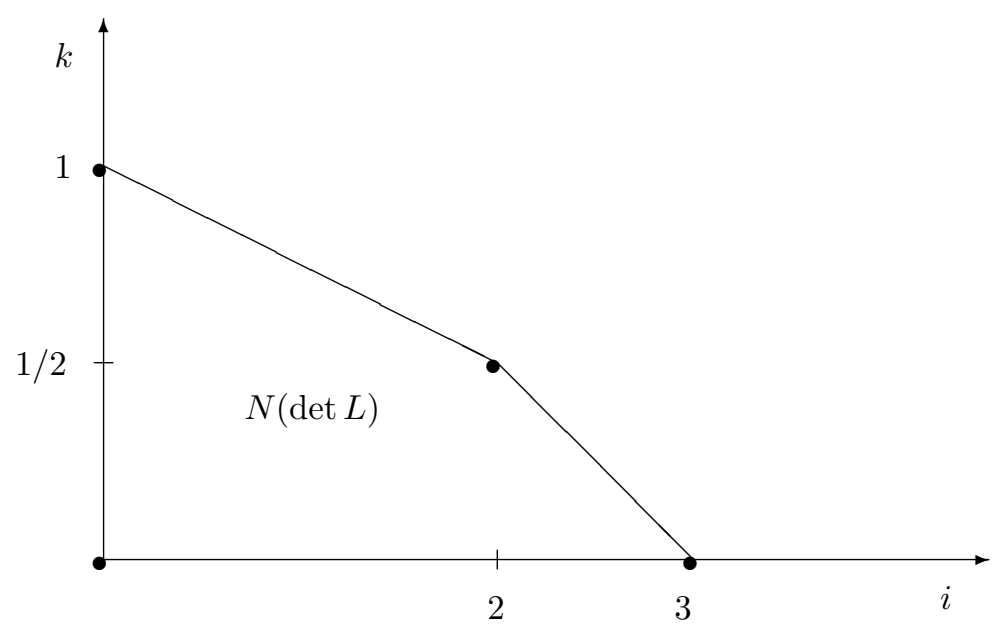

Figure 1. The Newton polygon for the Stefan problem

and

$$
\sum_{j=1}^{2}\left(s_{j}(\rho)+t_{j}(\rho)\right)= \begin{cases}\rho, & \rho \geq 4, \\ 2+\frac{\rho}{2}, & 4 \geq \rho \geq 2, \\ 3, & \rho \leq 2 .\end{cases}
$$

For all $\rho$, the right-hand side of the last equality coincides with $\operatorname{ord}_{\rho} \operatorname{det} L\left(\tau, \xi^{\prime}\right)$. From this we see that the matrix (2-2) satisfies all conditions of Definition 3.2 and the Stefan problem (1-4) is N-parabolic.

3.6. N-parabolicity of the Cahn-Hilliard equation with dynamic boundary conditions. Let us consider the Cahn-Hilliard equation with dynamic boundary conditions (1-5). The Lopatinskii matrix for this problem is given by (2-3) and its determinant equals to

$$
\operatorname{det} L\left(\xi^{\prime}, \tau\right)=i\left(i \tau+\left|\xi^{\prime}\right|^{2}\right)\left(z_{1}^{2}+z_{2}^{2}+z_{1} z_{2}+\left|\xi^{\prime}\right|^{2}\right)-z_{1} z_{2}\left(z_{1}+z_{2}\right) .
$$

We will need an elementary lemma.

Lemma 3.5. Let $z_{1}=z_{1}\left(\xi^{\prime}, \tau\right)$ and $z_{2}=z_{2}\left(\xi^{\prime}, \tau\right)$ be the roots of the equation

$$
\left(z^{2}+\left|\xi^{\prime}\right|^{2}\right)^{2}+i \tau=0
$$

with $\operatorname{Im} z_{1}>0, \operatorname{Im} z_{2}>0$ where $\operatorname{Im} \tau \leq 0$. Then

$$
\left.\left.\left|z_{1}^{2}+z_{2}^{2}+z_{1} z_{2}+\right| \xi^{\prime}\right|^{2}|\approx| \xi^{\prime}\right|^{2}+|\tau|^{1 / 2}
$$

Proof. The proof is based on two elementary observations.

(i) With appropriate numbering, we have

$$
z_{1}^{2}+\left|\xi^{\prime}\right|^{2}=\sqrt{-i \tau}, z_{2}^{2}+\left|\xi^{\prime}\right|^{2}=-\sqrt{-i \tau} .
$$

(ii) The product of $z_{1}$ and $z_{2}$ is equal to $z_{1} z_{2}=-\sqrt{\left|\xi^{\prime}\right|^{4}+i \tau}$.

From (i) we directly obtain

$$
z_{1}^{2}+z_{2}^{2}+\left|\xi^{\prime}\right|^{2}=-\left|\xi^{\prime}\right|^{2} .
$$


Now we apply (ii) to get

$$
\left.\left|z_{1}^{2}+z_{2}^{2}+z_{1} z_{2}+\right| \xi^{\prime}\right|^{2}|=|-|\xi|^{2}-\sqrt{|\xi|^{4}+i \tau} \mid \leq C_{2}\left(|\xi|^{2}+|\tau|^{1 / 2}\right)
$$

for $\operatorname{Im} \tau \leq 0$. In the same way we have

$$
\left.|| \xi\right|^{2}+\sqrt{|\xi|^{4}+i \tau} \mid \geq C_{1}\left(|\xi|^{2}+|\tau|^{1 / 2}\right) .
$$

We still have to prove (i) and (ii). For this we use the explicit formula for the root of a complex number $A+i B$

$$
\sqrt{A+i B}= \pm\left(\sqrt{\frac{A+\sqrt{A^{2}+B^{2}}}{2}}+i \sqrt{\frac{-A+\sqrt{A^{2}+B^{2}}}{2}}\right) .
$$

According to this formula, the sign "+" selects the root with positive imaginary part, and the sign "-" selects the root with negative imaginary part.

The equation $\left(z^{2}+\left|\xi^{\prime}\right|^{2}\right)^{2}+i \tau=0$ has the four roots

$$
z_{1,2,3,4}= \pm \sqrt{-\left|\xi^{\prime}\right|^{2} \pm \sqrt{-i \tau}}
$$

The roots with positive imaginary part are given by

$$
z_{1}=\sqrt{-\left|\xi^{\prime}\right|^{2}+\sqrt{-i \tau}}, z_{2}=\sqrt{-\left|\xi^{\prime}\right|^{2}-\sqrt{-i \tau}} .
$$

From this we get (i). To see (ii), we write

$$
z_{1}=i \sqrt{\left|\xi^{\prime}\right|^{2}-\sqrt{-i \tau}} \text { and } z_{2}=i \sqrt{\left|\xi^{\prime}\right|^{2}+\sqrt{-i \tau}}
$$

and obtain $z_{1} z_{2}=-\sqrt{\left|\xi^{\prime}\right|^{4}+i \tau}$.

From this lemma we obtain for large $-\operatorname{Im} \tau$

$$
\begin{aligned}
\left|\operatorname{det} L\left(\tau, \xi^{\prime}\right)\right| & \geq C_{1}\left(1+|\tau|^{1 / 2}+\left|\xi^{\prime}\right|^{2}\right)\left(1+|\tau|^{1 / 2}+\left|\xi^{\prime}\right|^{2} \mid\right)-C_{2}\left(|\tau|^{3 / 4}+\left|\xi^{\prime}\right|^{3}\right) \\
& \geq C_{3}\left(1+|\tau|^{3 / 2}+\left|\xi^{\prime}\right|^{2}|\tau|+\left|\xi^{\prime}\right|^{4}\right)
\end{aligned}
$$

and

$$
\left|\operatorname{det} L\left(\xi^{\prime}, \tau\right)\right| \approx 1+|\tau|^{3 / 2}+\left|\xi^{\prime}\right|^{2}|\tau|+\left|\xi^{\prime}\right|^{4} .
$$

Therefore, the Newton polygon of $\operatorname{det} L\left(\xi^{\prime}, \tau\right)$ has the vertices $(0,0),\left(0, \frac{3}{2}\right),(2,1)$ and $(4,0)$ (see Figure 2).

For every $\rho>0$, we define $s_{j}(\rho)$ and $t_{k}(\rho)(j, k=1,2)$ by $s_{2}(\rho)=0, t_{2}(\rho)=0$, and

$$
\begin{array}{ll}
s_{1}(\rho)=2, t_{1}(\rho)=2 & \text { if } \rho \leq 2, \\
s_{1}(\rho)=2, t_{1}(\rho)=\rho & \text { if } 2 \leq \rho \leq 4, \\
s_{1}(\rho)=\frac{\rho}{2}, t_{1}(\rho)=\rho & \text { if } \rho \geq 4 .
\end{array}
$$

Then it is easy to check that

$$
\operatorname{ord}_{\rho} L_{j k}\left(\xi^{\prime}, \tau\right) \leq s_{j}(\rho)+t_{k}(\rho) \quad(j, k=1,2)
$$

and

$$
\sum_{j=1}^{2}\left(s_{j}(\rho)+t_{j}(\rho)\right)=\operatorname{ord}_{\rho} L\left(\xi^{\prime}, \tau\right) .
$$

From this we see that the Cahn-Hilliard problem (1-5) is N-parabolic. 


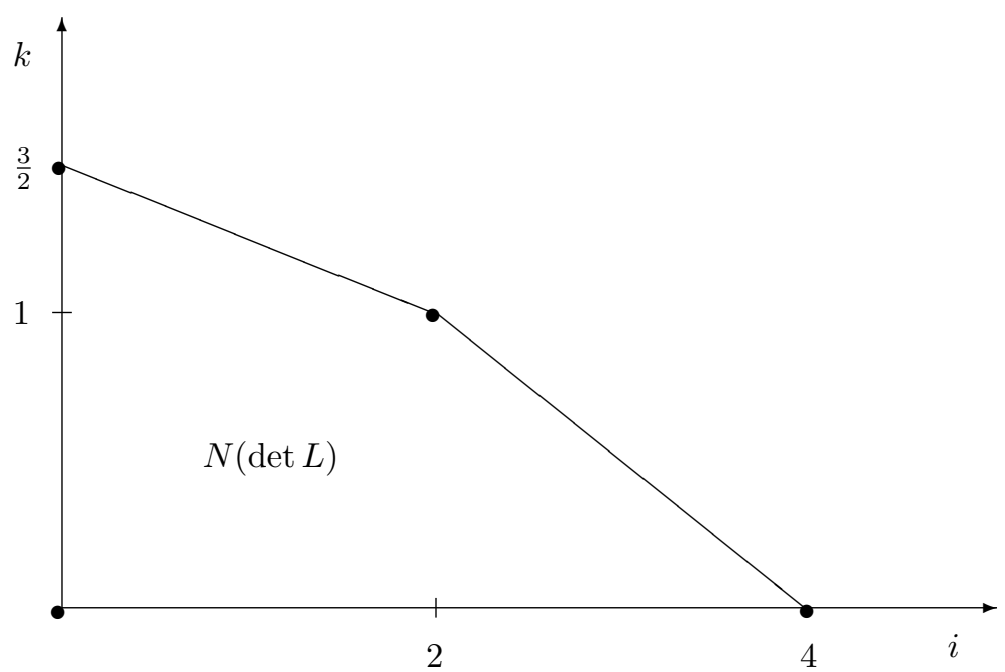

Figure 2. The Newton polygon for the Cahn-Hilliard equation

\section{Functional spaces}

In this short section we will introduce the functional spaces in which we will prove unique solvability of N-parabolic boundary value problems. For nonstationary problems, as a rule, the corresponding Sobolev spaces are defined by pseudodifferential operators with symbols which are analytic in the co-variable $\tau$ dual to the time variable. However, the main construction of [12] used below does not permit to construct symbols holomorphic in $\tau$. In the situation considered in the present paper, the systems are defined by the Lopatinskii matrix which is holomorphic in $\tau$. Thus we will be able to prove unique solvability on the half-line in the framework of spaces defined below.

4.1. Functional spaces $H^{\chi}$. At first we recall the known definition of functional spaces defined by means of pseudodifferential operators (see [13]).

Let $\mathbb{C}_{-}:=\{z \in \mathbb{C}: \operatorname{Im} z<0\}$. Denote by $\mathcal{F}\left(\mathbb{R}^{n} \times \mathbb{C}_{-}\right)=\mathcal{F}$ the set of all functions $\chi: \mathbb{R}^{n} \times \mathbb{C}_{-} \rightarrow \mathbb{C}$ which do not vanish and for which

$$
\left|\frac{\chi\left(\xi^{1}, \sigma^{1}\right)}{\chi\left(\xi^{2}, \sigma^{2}\right)}\right| \leq C\left(1+\left|\xi^{1}-\xi^{2}\right|+\left|\sigma^{1}-\sigma^{2}\right|\right)^{M(\chi)} \quad\left(\xi^{1,2} \in \mathbb{R}^{n}, \sigma^{1,2} \in \mathbb{C}_{-}\right)
$$

holds with some positive constant $M(\chi)$. We set

$$
H^{\chi}\left(\mathbb{R}^{n+1}\right):=\left\{u(x, t) \in H^{-\infty}\left(\mathbb{R}^{n+1}\right) \mid \chi\left(D_{x}, D_{t}\right) u \in L_{2}\left(\mathbb{R}^{n+1}\right)\right\} .
$$

This space is endowed with the norm

$$
\left\|u, H^{\chi}\left(\mathbb{R}^{n+1}\right)\right\|:=\left\|\chi\left(D_{x}, D_{t}\right) u, L_{2}\left(\mathbb{R}^{n+1}\right)\right\| .
$$

Replacing the symbol $\chi(\xi, \sigma)$ by an equivalent symbol $\chi^{\prime}(\xi, \sigma) \approx \chi(\xi, \sigma)$, we obtain an equivalent norm in $H^{\chi}\left(\mathbb{R}^{n+1}\right)$. In the case $\chi(\xi, \sigma) \approx 1+|\xi|^{r}+|\sigma|^{s}$ we will also write $H^{r, s}\left(\mathbb{R}^{n+1}\right)$ instead of $H^{\chi}\left(\mathbb{R}^{n+1}\right)$.

In a standard way the space $H^{\chi}\left(\mathbb{R}_{+}^{n} \times \mathbb{R}\right)$ is defined as the factor space of $H^{\chi}\left(\mathbb{R}^{n+1}\right)$ modulo the subspace of all distributions in $H^{\chi}\left(\mathbb{R}^{n+1}\right)$ whose support 
belongs to $\mathbb{R}_{-}^{n} \times \mathbb{R}:=\left\{(x, t) \in \mathbb{R}^{n} \times \mathbb{R}: x_{n}<0\right\}$. This space is endowed with the standard factor-norm.

For $\chi \in \mathcal{F}$ and $\gamma<0$ we set $\chi_{\gamma}(\xi, \sigma):=\chi(\xi, \sigma+i \gamma)$. For $\gamma, \gamma^{\prime} \in \mathcal{F}$ we have

$$
\left|\chi_{\gamma}(\xi, \sigma) / \chi_{\gamma^{\prime}}(\xi, \sigma)\right| \leq C\left(1+\left|\gamma-\gamma^{\prime}\right|\right)^{M(\chi)} \text {. }
$$

Varying the parameter $\gamma$, we obtain a family of norms $\left\|\chi_{\gamma}\left(D_{x}, D_{t}\right) u, L_{2}\left(\mathbb{R}^{n+1}\right)\right\|$ in the space $H^{\chi}\left(\mathbb{R}^{n+1}\right)$ which are equivalent.

4.2. Functional spaces $H_{[\gamma]}^{\chi}$ with exponential weight. For $\chi \in \mathcal{F}\left(\mathbb{R}^{n} \times \mathbb{C}_{-}\right)$ and $\gamma<0$ we define $H_{[\gamma]}^{\chi}\left(\mathbb{R}^{n} \times \mathbb{R}\right)$ as the space of such distributions $u(x, t)$ that $\exp (\gamma t) u(x, t) \in H^{\chi}\left(\mathbb{R}^{n+1}\right)$. We endow this space with the norm

$$
\left\|u, H_{[\gamma]}^{\chi}\left(\mathbb{R}^{n} \times \mathbb{R}\right)\right\|=\left\|\chi_{\gamma}\left(D_{x}, D_{t}\right)(\exp (\gamma t) u), L_{2}\left(\mathbb{R}^{n+1}\right)\right\| .
$$

For a function $u(x, t)$ in $\mathbb{R}^{n} \times \mathbb{R}$ denote by $\hat{u}(\xi, \tau)$ the complex Fourier transform or, equivalently, the real Fourier transform of $\exp (\gamma t) u(x, t)$ where $\xi \in \mathbb{R}^{n}$ and $\tau=\sigma+i \gamma \in \mathbb{C}$. For a weight function $\chi \in \mathcal{F}\left(\mathbb{R}^{n} \times \mathbb{C}_{+}\right)$and $\gamma<0$ we obtain the relation

$$
\left\|u, H_{[\gamma]}^{\chi}\left(\mathbb{R}^{n} \times \mathbb{R}\right)\right\|=\left(\int_{\operatorname{Im} \tau=\gamma} \int_{\mathbb{R}^{n}} \chi(\xi, \tau)^{2}|\hat{u}(\xi, \tau)|^{2} d \xi d \tau\right)^{1 / 2} .
$$

As above, in the case $\chi(\xi, \tau) \approx 1+|\xi|^{r}+|\tau|^{s}$ we will write $H_{[\gamma]}^{r, s}\left(\mathbb{R}^{n} \times \mathbb{R}\right)$.

In a standard way we denote by $H_{[\gamma]}^{\chi}\left(\mathbb{R}_{+}^{n} \times \mathbb{R}\right)$ the factor space of $H_{[\gamma]}^{\chi}\left(\mathbb{R}^{n} \times \mathbb{R}\right)$ modulo the subspace of all distributions in $H_{[\gamma]}^{\chi}\left(\mathbb{R}^{n} \times \mathbb{R}\right)$ whose support belongs to $\mathbb{R}_{-}^{n} \times \mathbb{R}$. This space is endowed with standard factor-norm. We will write $H_{[\gamma]}^{\chi}\left(\mathbb{R}^{n-1} \times \mathbb{R}\right)$ for the corresponding space of functions defined on the hyperplane $\left\{x_{n}=0,\left(x^{\prime}, t\right) \in \mathbb{R}^{n-1} \times \mathbb{R}\right\}$.

We denote by $H_{[\gamma]+}^{\chi}\left(\mathbb{R}^{n} \times \mathbb{R}\right), H_{[\gamma]+}^{\chi}\left(\mathbb{R}_{+}^{n} \times \mathbb{R}\right)$ etc. the subspaces of the above spaces consisting of distributions vanishing for $t \leq 0$.

Remark 4.1. a) From the definitions we see that

$$
\phi:=e^{-\gamma t} \chi\left(D_{x}, D_{t}+i \gamma\right) e^{\gamma t} u \in L_{2[\gamma]}\left(\mathbb{R}^{n+1}\right) \quad \text { if } \quad u \in H_{[\gamma]}^{\chi}\left(\mathbb{R}^{n} \times \mathbb{R}\right) .
$$

Therefore, $u \in H_{[\gamma]}^{\chi}\left(\mathbb{R}^{n} \times \mathbb{R}\right)$ can be represented as

$$
u=e^{-\gamma t} \chi^{-1}\left(D_{x}, D_{t}+i \gamma\right) e^{\gamma t} \phi, \quad \phi \in L_{2[\gamma]}\left(\mathbb{R}^{n+1}\right) .
$$

In other words the map

$$
H_{[\gamma]}^{\chi}\left(\mathbb{R}^{n} \times \mathbb{R}\right) \longrightarrow L_{2[\gamma]}\left(\mathbb{R}^{n+1}\right), \quad u \longmapsto \phi:=e^{-\gamma t} \chi\left(D_{x}, D_{t}+i \gamma\right) e^{\gamma t} u
$$

is an isomorphism of Sobolev spaces. Unfortunately the restriction of the isomorphism (4-5) to the subspace of functions with support on a half-axis is not an isomorphism.

b) Let $\mathcal{F}_{\text {hol }}$ be the space of weight functions from $\mathcal{F}$ which are holomorphic for $\operatorname{Im} \tau<0$. In the case $\chi \in \mathcal{F}_{\text {hol }}$ all the statements of Remark a) hold if we replace $H_{[\gamma]}^{\chi}\left(\mathbb{R}^{n} \times \mathbb{R}\right)$ and $L_{2[\gamma]}\left(\mathbb{R}^{n+1}\right)$ by $H_{[\gamma]+}^{\chi}\left(\mathbb{R}^{n} \times \mathbb{R}\right)$ and $L_{2[\gamma]+}\left(\mathbb{R}^{n+1}\right)$, respectively. In particular, the map

$$
H_{[\gamma]+}^{\chi}\left(\mathbb{R}^{n} \times \mathbb{R}\right) \longrightarrow L_{2[\gamma]+}\left(\mathbb{R}^{n+1}\right), \quad u \longmapsto \phi:=e^{-\gamma t} \chi\left(D_{x}, D_{t}+i \gamma\right) e^{\gamma t} u
$$

is an isomorphism. This yields the inclusions $H_{[\gamma]+}^{\chi} \subset H_{[\rho]+}^{\chi}$ for all $\rho<\gamma$ (see the detailed exposition in [6]). 


\subsection{Functional spaces $H_{[\gamma]}^{\chi}$ connected with the Newton polygon. Let $\Gamma$}

be a convex polygon in the positive quadrant with $(0,0)$ as one vertex and the coordinate lines as two edges. Denote by $\left(p_{0}, q_{0}\right), \ldots,\left(p_{J+1}, q_{J+1}\right)$ the vertices of polygon $\Gamma$, starting with $(0,0)$ and indexed in clockwise direction. Define

$$
\Xi_{\Gamma}(\xi, \tau):=1+\sum_{j=1}^{J+1}|\xi|^{p_{j}}|\tau|^{q_{j}} .
$$

We denote by $H_{[\gamma]}^{(\Gamma)}$ the space $H_{[\gamma]}^{\chi}$ with $\chi(\xi, \tau) \approx \Xi_{\Gamma}(\xi, \tau)$ for $\operatorname{Im} \tau \leq 0$.

Lemma 4.2. For a given polygon $\Gamma$ there exists a weight function $\lambda_{\Gamma}(\xi, \tau) \in \mathcal{F}_{\text {hol }}$ such that $\lambda_{\Gamma}(\xi, \tau) \approx \Xi_{\Gamma}(\xi, \tau)$ for $\operatorname{Im} \tau \leq 0$.

Proof. Using the argument of [5], Chapter I, Subsection 1.3, it is easy to prove that

$$
\Xi_{\Gamma}(\xi, \tau) \approx \prod_{j=1}^{J}\left(1+|\xi|^{p_{j+1}-p_{j}}+|\tau|^{q_{j}-q_{j+1}}\right) .
$$

Now it remains to note that for $\operatorname{Im} \tau \leq 0$

$$
1+|\xi|^{p_{j+1}-p_{j}}+|\tau|^{q_{j}-q_{j+1}} \approx\left(1+|\xi|^{r_{j}}+i \tau\right)^{q_{j}-q_{j+1}},
$$

where

$$
r_{j}=\frac{p_{j+1}-p_{j}}{q_{j}-q_{j+1}}
$$

\section{Solvability of N-PARABolic Boundary Value Problems.}

In this section we discuss unique solvability of the model problem (1-1)-(1-2) in the half-space by reducing it to the system $(3-8)$ or (3-10) on the boundary. We will obtain unique solvability and two-sided a priori estimates in the weighted $L_{2^{-}}$ Sobolev spaces defined above, provided some additional conditions on the weight function hold. We will see in Section 6 that for the crystallization problems considered in this paper these additional conditions are satisfied.

We will prove the main solvability result in three steps: first we will consider the system defined by the Lopatinskii matrix, then consider (1-1)-(1-2) with $f=0$, and finally we will treat the general case.

5.1. Solvability of the system in $\mathbb{R}^{n-1} \times \mathbb{R}$ defined by the Lopatinskii matrix of an N-parabolic problem. We will study the systems (3-8) (or (3-10)). They have the same structure and we use the same notation. The number of unknown functions will be denoted by $M$, where either $M=m$ or $M=m+\kappa$. Using the technique of [12], we can prove the following main result.

Theorem 5.1. Suppose the problem (1-1)-(1-2) is $N$-parabolic, and let $L\left(\xi^{\prime}, \tau\right)$ be the Lopatinskii matrix of the problem. Then there exist weight functions $\mu_{j}, \nu_{j} \in$ $\mathcal{F}\left(\mathbb{R}^{n-1} \times \mathbb{C}_{-}\right) \quad(j=1, \ldots, M)$ and $\gamma_{0}<0$ such that the following statements hold.

(i) Let $\chi \in \mathcal{F}\left(\mathbb{R}^{n-1} \times \mathbb{C}_{-}\right)$and $\gamma<\gamma_{0}$. For every

$$
g=\left(g_{1}, \ldots, g_{M}\right)^{T} \in \prod_{k=1}^{M} H_{[\gamma]}^{\chi \cdot \nu_{k}^{-1}}\left(\mathbb{R}^{n-1} \times \mathbb{R}\right)
$$


the system

$$
\sum_{k=1}^{M} L_{j k}\left(D_{x^{\prime}}, D_{t}\right) h_{k}=g_{j} \quad(j=1, \ldots, M)
$$

has a unique solution $h=\left(h_{1}, \ldots, h_{M}\right)^{T} \in \prod_{j=1}^{M} H_{[\gamma]}^{\chi \cdot \mu_{j}}\left(\mathbb{R}^{n-1} \times \mathbb{R}\right)$, and the estimate

$$
\begin{gathered}
C^{-1} \sum_{j=1}^{M}\left\|h_{j}, H_{[\gamma]}^{\chi \cdot \mu_{j}}\left(\mathbb{R}^{n-1} \times \mathbb{R}\right)\right\| \leq \sum_{k=1}^{M}\left\|g_{k}, H_{[\gamma]}^{\chi \cdot \nu_{k}^{-1}}\left(\mathbb{R}^{n-1} \times \mathbb{R}\right)\right\| \\
\leq C \sum_{j=1}^{M}\left\|h_{j}, H_{[\gamma]}^{\chi \cdot \mu_{j}}\left(\mathbb{R}^{n-1} \times \mathbb{R}\right)\right\|
\end{gathered}
$$

holds.

(ii) Let $\chi \in \mathcal{F}\left(\mathbb{R}^{n-1} \times \mathbb{C}_{-}\right)$and $\gamma<\gamma_{0}$. For arbitrary

$$
g=\left(g_{1}, \ldots, g_{M}\right)^{T} \in \prod_{k=1}^{M} H_{[\gamma]+}^{\chi \cdot \nu_{k}^{-1}}\left(\mathbb{R}^{n-1} \times \mathbb{R}\right)
$$

equation (5-1) has a unique solution $h=\left(h_{1}, \ldots, h_{M}\right)^{T} \in \prod_{j=1}^{M} H_{[\gamma]+}^{\chi \cdot \mu_{j}}\left(\mathbb{R}^{n-1} \times \mathbb{R}\right)$, and for this solution estimate (5-2) holds.

The proof of the theorem is based on a statement which was essentially proved in [12]. We will comment on the proof of the following proposition in Subsection 5.4.

Proposition 5.2. Suppose the Lopatinskii matrix $L\left(\xi^{\prime}, \tau\right)=\left(L_{j k}\left(\xi^{\prime}, \tau\right)\right)_{j, k=1, \ldots, M}$ is $N$-parabolic. Then there exist weight functions $\mu_{j}, \nu_{j} \in \mathcal{F}(j=1, \ldots, M)$ and $\gamma_{0}<0$ such that

$$
\left|L_{j k}\left(\xi^{\prime}, \tau\right)\right| \leq C\left|\mu_{j}\left(\xi^{\prime}, \tau\right)\right| \cdot\left|\nu_{k}\left(\xi^{\prime}, \tau\right)\right| \quad(j, k=1, \ldots, M)
$$

and

$$
\left|\operatorname{det} L\left(\xi^{\prime}, \tau\right)\right| \geq C \prod_{j=1}^{M}\left|\mu_{j}\left(\xi^{\prime}, \tau\right)\right| \cdot\left|\nu_{j}\left(\xi^{\prime}, \tau\right)\right| \quad\left(\operatorname{Im} \tau \leq \gamma_{0}\right)
$$

Proof of Theorem 5.1. Denote by $G\left(\xi^{\prime}, \tau\right)=\left(G_{j k}\left(\xi^{\prime}, \tau\right)\right)_{j, k=1, \ldots, M}$ the inverse of the matrix $L\left(\xi^{\prime}, \tau\right)$. As it was remarked in [12], the estimates (5-3) and (5-4) induce estimates for the elements of $G$ :

$$
\left|G_{j k}\left(\xi^{\prime}, \tau\right)\right| \leq C\left|\mu_{j}^{-1}\left(\xi^{\prime}, \tau\right)\right| \cdot\left|\nu_{k}^{-1}\left(\xi^{\prime}, \tau\right)\right| .
$$

Since

$$
h_{j}=\sum_{k=1}^{M} G_{j k}\left(D_{x^{\prime}}, D_{t}\right) g_{k}
$$

is the solution of (5-1), these estimates easily imply statement (i).

To prove (ii) it is enough to show that the elements of $G$ are holomorphic in $\tau$ for $\operatorname{Im} \tau<0$. According to Subsection 2.5, the symbols $L_{j k}\left(\xi^{\prime}, \tau\right)$ are holomorphic in $\tau$ for $\operatorname{Im} \tau<0$, hence $\operatorname{det} L\left(\xi^{\prime}, \tau\right)$ has the same property. From (5-4) we know that the determinant does not vanish and $\left(\operatorname{det} L\left(\xi^{\prime}, \tau\right)\right)^{-1}$ also holomorphic in $\tau$ for $\operatorname{Im} \tau<0$. Since the elements $G_{j k}\left(\xi^{\prime}, \tau\right)$ are polynomials in $L_{j k}\left(\xi^{\prime}, \tau\right)$ divided by $\operatorname{det} L\left(\xi^{\prime}, \tau\right)$, they are also holomorphic. 
5.2. Solvability of problem (1-1)-(1-2) in the case $f \equiv 0$. Now we apply Theorem 5.1 to obtain the solvability of the parabolic problem in the half-space. The order of the $2 b$-parabolic equation (2.1) is equal to $2 m$, so it is natural to search the solution $u(x, t)$ of $(2.1)$ in the space $H_{[\gamma]+}^{r, \frac{r}{2 b}}\left(\mathbb{R}_{+}^{n} \times \mathbb{R}\right)$, where $r \geq 2 m$ is such that boundary conditions (1-2) are defined in $H_{[\gamma]}^{r, \frac{r}{2 b}}$.

According to Subsection 3.4, in the case $f \equiv 0$ the unknown function $u$ can be found as solution of the homogeneous parabolic equation with the Dirichlet boundary conditions

$$
\gamma_{j-1} u\left(x^{\prime}, t\right):=\left.D_{n}^{j-1} u(x, t)\right|_{x_{n}=0}=h_{j}\left(x^{\prime}, t\right) \quad(j=1, \ldots, m)
$$

where $\left(h_{1}, \ldots, h_{M}\right)^{T}$ is the solution of the system (5-1).

If our problem is N-parabolic then there exist the weight functions from Proposition 5.2 such that the statement of Theorem 5.1 (ii) holds. To obtain a solution $u(x, t) \in H_{[\gamma]+}^{r, \frac{r}{2 b}}\left(\mathbb{R}_{+}^{n} \times \mathbb{R}\right)$ we must demand the Dirichlet data to belong to the trace space of $H_{[\gamma]+}^{r, \frac{r}{2 b}}\left(\mathbb{R}_{+}^{n} \times \mathbb{R}\right)$ :

$$
h_{j}\left(x^{\prime}, t\right)=\gamma_{j-1} u\left(x^{\prime}, t\right) \in H_{[\gamma]+}^{r-j+\frac{1}{2}, \frac{r}{2 b}-\frac{j}{2 b}+\frac{1}{4 b}}\left(\mathbb{R}^{n-1} \times \mathbb{R}\right) \quad(j=1, \ldots, m) .
$$

On the other side, according to Theorem 5.1 (ii), if $g_{k} \in H_{[\gamma]+}^{\chi \cdot \nu_{k}^{-1}}\left(\mathbb{R}^{n-1} \times \mathbb{R}\right)$ for $k=$ $1, \ldots, M$ then $h_{j} \in H_{[\gamma]+}^{\chi \cdot \mu_{j}}\left(\mathbb{R}^{n-1} \times \mathbb{R}\right)$ and the estimate (5-2) holds. Therefore, for conditions (5-6) to be valid we must suppose that following continuous embeddings

$$
H_{[\gamma]+}^{\chi \cdot \mu_{j}}\left(\mathbb{R}^{n-1} \times \mathbb{R}\right) \subset H_{[\gamma]+}^{r-j+\frac{1}{2}, \frac{r}{2 b}-\frac{j}{2 b}+\frac{1}{4 b}}\left(\mathbb{R}^{n-1} \times \mathbb{R}\right) \quad(j=1, \ldots, m)
$$

hold. These embeddings will take place if and only if

$$
\chi\left(\xi^{\prime}, \tau\right) \geq C \frac{\left(1+\left|\xi^{\prime}\right|+|\tau|^{\frac{1}{2 b}}\right)^{r-j+\frac{1}{2}}}{\mu_{j}\left(\xi^{\prime}, \tau\right)} \quad(j=1, \ldots, m) .
$$

As a result we proved the

Theorem 5.3. Suppose problem (1-1)-(1-2) is $N$-parabolic. Let $\mu_{j}, \nu_{j} \in \mathcal{F}\left(\mathbb{R}^{n-1} \times\right.$ $\left.\mathbb{C}_{-}\right)(j=1, \ldots, M)$ be weight functions as in Proposition 5.2. Then there exists a $\gamma_{0}<0$ such that for each $r \geq 2 m$ and each $\chi \in \mathcal{F}\left(\mathbb{R}^{n-1} \times \mathbb{C}_{-}\right)$satisfying (5-8) the following statement holds.

For each

$$
\left(g_{1}, \ldots, g_{M}\right)^{T} \in \prod_{k=1}^{M} H_{[\gamma]+}^{\chi \cdot \nu_{k}^{-1}}\left(\mathbb{R}^{n-1} \times \mathbb{R}\right)
$$

the boundary value problem (1-1)-(1-2) with $f=0$ has a unique solution

$$
\left(u, \sigma_{1}, \ldots, \sigma_{\kappa}\right) \in H_{[\gamma]+}^{r, \frac{r}{2 b}}\left(\mathbb{R}_{+}^{n} \times \mathbb{R}\right) \times \prod_{j=1}^{\kappa} H_{[\gamma]+}^{\chi \cdot \mu_{j+m}}\left(\mathbb{R}^{n-1} \times \mathbb{R}\right) .
$$

Moreover the traces $\gamma_{0} u, \ldots, \gamma_{m-1} u$ have the additional smoothness

$$
\gamma_{j-1} u \in H_{[\gamma]+}^{\chi \cdot \mu_{j}}\left(\mathbb{R}^{n-1} \times \mathbb{R}\right) \quad(j=1, \ldots, m),
$$


and the two-sided estimate

$$
\begin{aligned}
& \left\|u, H_{[\gamma]}^{r, \frac{r}{2 b}}\left(\mathbb{R}_{+}^{n} \times \mathbb{R}\right)\right\|+\sum_{j=1}^{\kappa}\left\|\sigma_{j}, H_{[\gamma]}^{\chi \cdot \mu_{j+m}}\left(\mathbb{R}^{n-1} \times \mathbb{R}\right)\right\| \\
& \quad+\sum_{j=1}^{m}\left\|\gamma_{j-1} u, H_{[\gamma]}^{\chi \cdot \mu_{j}}\left(\mathbb{R}^{n-1} \times \mathbb{R}\right)\right\| \approx \sum_{k=1}^{M}\left\|g_{k}, H_{[\gamma]}^{\chi \cdot \nu_{k}^{-1}}\left(\mathbb{R}^{n-1} \times \mathbb{R}\right)\right\|
\end{aligned}
$$

holds.

5.3. Solvability of problem (1-1)-(1-2) in the general case. As in the preceding subsection, we want to find the solution $u$ in $H_{[\gamma]+}^{r, \frac{r}{2 b}}\left(\mathbb{R}_{+}^{n} \times \mathbb{R}\right)$ with sufficiently large $r$. In principle, the right hand side $f$ should belong to $H_{[\gamma]+}^{r-2 m, \frac{r-2 m}{2 b}}\left(\mathbb{R}_{+}^{n} \times \mathbb{R}\right)$.

However, in general such smoothness of $f$ does not guarantee the necessary smoothness (and even existence) of the terms in the boundary conditions. So we have to suppose that $f$ has an additional smoothness, $f \in H_{[\gamma]+}^{r^{\prime}-2 m, \frac{r^{\prime}-2 m}{2 b}}\left(\mathbb{R}_{+}^{n} \times \mathbb{R}\right)$, where $r^{\prime} \geq r$.

Theorem 5.4. Suppose (1-1)-(1-2) is $N$-parabolic and let $\mu_{j}, \nu_{j} \in \mathcal{F}\left(\mathbb{R}^{n-1} \times \mathbb{C}\right)$ be as Proposition 5.2. Suppose that for given $r \geq 2 m$ the weight function $\chi \in$ $\mathcal{F}\left(\mathbb{R}^{n-1} \times \mathbb{C}\right)$ satisfies conditions (5-8). Then there exists an $r^{\prime} \geq r$ and $a \gamma_{0}>0$ such that the following statements hold.

For each $f \in H_{[\gamma]+}^{r^{\prime}-2 m, \frac{r^{\prime}-2 m}{2 b}}\left(\mathbb{R}_{+}^{n} \times \mathbb{R}\right)$ and each

$$
\left(g_{1}, \ldots, g_{M}\right)^{T} \in \prod_{k=1}^{M} H_{[\gamma]+}^{\chi \cdot \nu_{k}^{-1}}\left(\mathbb{R}^{n-1} \times \mathbb{R}\right)
$$

the boundary value problem (1-1)-(1-2) has a unique solution

$$
\left(u, \sigma_{1}, \ldots, \sigma_{\kappa}\right) \in H_{[\gamma]+}^{r, \frac{r}{2 b}}\left(\mathbb{R}_{+}^{n} \times \mathbb{R}\right) \times \prod_{j=1}^{\kappa} H_{[\gamma]+}^{\chi \cdot \mu_{j+m}}\left(\mathbb{R}^{n-1} \times \mathbb{R}\right) .
$$

Moreover, the traces $\gamma_{0} u, \ldots, \gamma_{m-1} u$ have the additional smoothness

$$
\gamma_{j-1} u \in H_{[\gamma]+}^{\chi \cdot \mu_{j}}\left(\mathbb{R}^{n-1} \times \mathbb{R}\right) \quad(j=1, \ldots, m),
$$

and the a priori estimate

$$
\begin{aligned}
& \left\|u, H_{[\gamma]+}^{r, \frac{r}{2 b}}\left(\mathbb{R}_{+}^{n} \times \mathbb{R}\right)\right\|+\sum_{j=1}^{\kappa}\left\|\sigma_{j}, H_{[\gamma]}^{\chi \cdot \mu_{j+m}}\left(\mathbb{R}^{n-1} \times \mathbb{R}\right)\right\| \\
& \quad \leq C\left(\left\|f, H_{[\gamma]}^{r^{\prime}-2 m, \frac{r^{\prime}-2 m}{2 b}}\left(\mathbb{R}_{+}^{n} \times \mathbb{R}\right)\right\|+\sum_{k=1}^{M}\left\|g_{k}, H_{[\gamma]}^{\chi \cdot \nu_{k}^{-1}}\left(\mathbb{R}^{n-1} \times \mathbb{R}\right)\right\|\right)
\end{aligned}
$$

holds.

Proof. We will seek the solution $u$ in the form $u=u^{0}+u^{1}$ where $u^{0}$ is the solution of the Dirichlet problem with homogeneous boundary conditions

$$
\begin{aligned}
A\left(D_{x}, D_{t}\right) u^{0}(x, t) & =f(x, t) & & \text { in } \mathbb{R}_{+}^{n} \times \mathbb{R}, \\
\partial_{n}^{k-1} u^{0}\left(x^{\prime}, t\right) & =0 & & \text { in } \mathbb{R}^{n-1} \times \mathbb{R} \quad(k=1, \ldots, m), \\
u^{0}(x, t) & =f(x, t)=0 & & \text { for } t<0 .
\end{aligned}
$$


For every $f \in H_{[\gamma]+}^{r^{\prime}-2 m, \frac{r^{\prime}-2 m}{2 b}}\left(\mathbb{R}_{+}^{n} \times \mathbb{R}\right)$ this problem has a unique solution $u^{0}(x, t) \in$ $H_{[\gamma]+}^{r^{\prime}, \frac{r^{\prime}}{2 b}}\left(\mathbb{R}_{+}^{n} \times \mathbb{R}\right)$, and

$$
\left\|u^{0}, H_{[\gamma]+}^{r^{\prime}, \frac{r^{\prime}}{2 b}}\left(\mathbb{R}_{+}^{n} \times \mathbb{R}\right)\right\| \approx\left\|f, H_{[\gamma]+}^{r^{\prime}-2 m, \frac{r^{\prime}-2 m}{2 b}}\left(\mathbb{R}_{+}^{n} \times \mathbb{R}\right),\right\|
$$

The second function $u^{1}$ will be the solution of the homogeneous equation with inhomogeneous boundary data:

$$
\begin{aligned}
A\left(D_{x}, D_{t}\right) u^{1}(x, t) & =0 & & \text { in } \mathbb{R}_{+}^{n} \times \mathbb{R}, \\
B_{j}\left(D_{x}, D_{t}\right) u^{1}\left(x^{\prime}, t\right)+\sum_{k=1}^{\kappa} C_{j k}\left(D_{x^{\prime}}\right) \sigma_{k} & =\tilde{g}_{j}\left(x^{\prime}, t\right) & & \text { in } \mathbb{R}^{n-1} \times \mathbb{R}, \\
u^{1}(x, t) & =f(x, t)=0 & & \text { for } t<0 .
\end{aligned}
$$

Here

$$
\tilde{g}_{j}\left(x^{\prime}, t\right):=g_{j}\left(x^{\prime}, t\right)-B_{j}\left(D_{t}, D_{x}\right) u^{0}\left(x^{\prime}, t\right) \quad(j=1, \ldots, M) .
$$

If $r^{\prime}$ is large enough then

$$
B_{j}\left(D_{x}, D_{t}\right) u^{0}\left(x^{\prime}, t\right) \in H_{[\gamma]+}^{\chi \cdot \nu_{k}^{-1}}\left(\mathbb{R}^{n-1} \times \mathbb{R}\right), \quad j=1, \ldots, M,
$$

i.e. the functions $\tilde{g}_{j}\left(x^{\prime}, t\right)$ have the same smoothness as $g_{j}\left(x^{\prime}, t\right)$. Applying Theorem 5.3, we obtain the solution

$$
\left(u, \sigma_{1}, \ldots, \sigma_{\kappa}\right) \in H_{[\gamma]+}^{r, \frac{r}{2 b}}\left(\mathbb{R}_{+}^{n} \times \mathbb{R}\right) \times \prod_{j=1}^{\kappa} H_{[\gamma]+}^{\chi \cdot \mu_{j+m}}\left(\mathbb{R}^{n-1} \times \mathbb{R}\right) .
$$

As we shall see in the next section, there are N-parabolic problems where assumption (5-14) holds without assumption of the additional smoothness of the right-hand side $f$. In this case we obtain a two-sided estimate.

Definition 5.5. The number $r \geq 2 m$, the weight functions $\mu_{j}, \nu_{j} \in \mathcal{F}\left(\mathbb{R}^{n-1} \times \mathbb{C}_{-}\right)$ from Proposition 5.2, and a weight function $\chi \in \mathcal{F}$ satisfying (5-8) are said to be compatible with the boundary conditions if the operators

$\gamma_{0} B_{k}\left(D_{x}, D_{t}\right):\left\{u \in H_{[\gamma]+}^{r, \frac{r}{2 b}}\left(\mathbb{R}_{+}^{n} \times \mathbb{R}\right): \gamma_{0} u=\cdots=\gamma_{m-1} u=0\right\} \rightarrow H_{[\gamma]+}^{\chi \cdot \nu_{k}^{-1}}\left(\mathbb{R}^{n-1} \times \mathbb{R}\right)$ are continuous for $k=1, \ldots, M$.

Theorem 5.6. Suppose (1-1)-(1-2) is $N$-parabolic and let $\mu_{j}, \nu_{j} \in \mathcal{F}\left(\mathbb{R}^{n-1} \times \mathbb{C}\right)$ be as Proposition 5.2. Let $r \geq 2 m$ and $\chi \in \mathcal{F}\left(\mathbb{R}^{n-1} \times \mathbb{C}\right)$ be a weight function satisfying (5-8), and assume that $r, \mu_{j}, \nu_{j}$ and $\chi$ are compatible to the boundary conditions. Then there exists a $\gamma_{0}>0$ such that the following statements hold.

For each $f \in H_{[\gamma]+}^{r-2 m, \frac{r-2 m}{2 b}}\left(\mathbb{R}_{+}^{n} \times \mathbb{R}\right)$ and each

$$
\left(g_{1}, \ldots, g_{M}\right)^{T} \in \prod_{k=1}^{M} H_{[\gamma]+}^{\chi \cdot \nu_{k}^{-1}}\left(\mathbb{R}^{n-1} \times \mathbb{R}\right)
$$

the boundary value problem (1-1)-(1-2) has a unique solution

$$
\left(u, \sigma_{1}, \ldots, \sigma_{\kappa}\right) \in H_{[\gamma]+}^{r, \frac{r}{2 b}}\left(\mathbb{R}_{+}^{n} \times \mathbb{R}\right) \times \prod_{j=1}^{\kappa} H_{[\gamma]+}^{\chi \cdot \mu_{j+m}}\left(\mathbb{R}^{n-1} \times \mathbb{R}\right) .
$$


Moreover, the traces $\gamma_{0} u, \ldots, \gamma_{m-1} u$ have the additional smoothness

$$
\gamma_{j-1} u \in H_{[\gamma]+}^{\chi \cdot \mu_{j}}\left(\mathbb{R}^{n-1} \times \mathbb{R}\right) \quad(j=1, \ldots, m),
$$

and the two-sided a priori estimate

$$
\begin{aligned}
\| u, H_{[\gamma]}^{r, \frac{r}{2 b}} & \left(\mathbb{R}_{+}^{n} \times \mathbb{R}\right)\left\|+\sum_{j=1}^{m}\right\| \gamma_{j-1} u, H_{[\gamma]}^{\chi \cdot \mu_{j}}\left(\mathbb{R}^{n-1} \times \mathbb{R}\right) \| \\
& +\sum_{j=1}^{\kappa}\left\|\sigma_{j}, H_{[\gamma]}^{\chi \cdot \mu_{j+m}}\left(\mathbb{R}^{n-1} \times \mathbb{R}\right)\right\| \\
& \approx\left\|f, H_{[\gamma]}^{r-2 m, \frac{r-2 m}{2 b}}\left(\mathbb{R}_{+}^{n} \times \mathbb{R}\right)\right\|+\sum_{k=1}^{M}\left\|g_{k}, H_{[\gamma]}^{\chi \cdot \nu_{k}^{-1}}\left(\mathbb{R}^{n-1} \times \mathbb{R}\right)\right\|
\end{aligned}
$$

holds.

Proof. Following the proof of Theorem 5.4, we write the solution $u$ in the form $u=u^{0}+u^{1}$ where $u^{0}$ is the solution of the homogeneous Dirichlet problem (5-11) and $u^{1}$ is the solution of the inhomogeneous problem (5-13).

As it was mentioned in the proof of Theorem 5.4, by classical parabolic theory (5-11) is uniquely solvable with solution $u^{0} \in H_{[\gamma]+}^{r, \frac{r}{2 b}}\left(\mathbb{R}_{+}^{n} \times \mathbb{R}\right)$ satisfying

$$
\left\|u^{0}, H_{[\gamma]}^{r, \frac{r}{2 b}}\left(\mathbb{R}_{+}^{n} \times \mathbb{R}\right)\right\| \approx\left\|f, H_{[\gamma]}^{r-2 m, \frac{r-2 m}{2 b}}\left(\mathbb{R}_{+}^{n} \times \mathbb{R}\right)\right\| .
$$

Again in (5-13) we have

$$
\tilde{g}_{j}\left(x^{\prime}, t\right)=g_{j}\left(x^{\prime}, t\right)-B_{j}\left(D_{t}, D_{x}\right) u^{0}\left(x^{\prime}, t\right) \quad(j=1, \ldots, M) .
$$

By the compatibility conditions, we obtain

$$
\gamma_{0} B_{j}\left(D_{x}, D_{t}\right) u^{0} \in H_{[\gamma]+}^{\chi \cdot \nu_{j}^{-1}}\left(\mathbb{R}^{n-1} \times \mathbb{R}\right) \quad(j=1, \ldots, M) .
$$

Thus

$$
\tilde{g}_{j}\left(x^{\prime}, t\right) \in H_{[\gamma]+}^{\chi \cdot \nu_{j}^{-1}}\left(\mathbb{R}^{n-1} \times \mathbb{R}\right) \quad(j=1, \ldots, M)
$$

and we can apply Theorem 5.3 to find a unique solution

$$
\begin{gathered}
\left(u^{1}, \sigma_{1}, \ldots, \sigma_{\kappa}\right) \in H_{[\gamma]+}^{r, \frac{r}{2 b}}\left(\mathbb{R}_{+}^{n} \times \mathbb{R}\right) \times \prod_{j=1}^{\kappa} H_{[\gamma]+}^{\chi \cdot \mu_{j+m}}\left(\mathbb{R}^{n-1} \times \mathbb{R}\right), \\
\gamma_{j-1} u^{1} \in H_{[\gamma]+}^{\chi \cdot \mu_{j}}\left(\mathbb{R}^{n-1} \times \mathbb{R}\right) \quad(j=1, \ldots, m),
\end{gathered}
$$

and the estimate

$$
\begin{aligned}
\left\|u^{1}, H_{[\gamma]}^{r, \frac{r}{2 b}}\left(\mathbb{R}_{+}^{n} \times \mathbb{R}\right)\right\| & +\sum_{j=1}^{\kappa}\left\|\sigma_{j}, H_{[\gamma]}^{\chi \cdot \mu_{j+m}}\left(\mathbb{R}^{n-1} \times \mathbb{R}\right)\right\| \\
& \approx \sum_{k=1}^{M}\left\|\tilde{g}_{k}, H_{[\gamma]}^{\chi \cdot \nu_{k}^{-1}}\left(\mathbb{R}^{n-1} \times \mathbb{R}\right)\right\|
\end{aligned}
$$

holds.

Now we show that the statement of the theorem for $u=u^{0}+u^{1}$ follows from (5-16) and (5-18). Indeed, obviously $u \in H_{[\gamma]+}^{r, \frac{r}{2 b}}\left(\mathbb{R}_{+}^{n} \times \mathbb{R}\right)$. Since $u^{0}$ has zero Dirichlet data

$$
\gamma_{j-1} u=\gamma_{j-1} u^{1} \in H_{[\gamma]+}^{\chi \cdot \mu_{j}}\left(\mathbb{R}^{n-1} \times \mathbb{R}\right) \quad(j=1, \ldots, m),
$$


and we have to check estimate (5-15).

We start with the left inequality and write

$$
\begin{aligned}
\left\|u, H_{[\gamma]}^{r, \frac{r}{2 b}}\left(\mathbb{R}_{+}^{n} \times \mathbb{R}\right)\right\|+\sum_{j=1}^{\kappa}\left\|\sigma_{j}, H_{[\gamma]}^{\chi \cdot \mu_{j+m}}\left(\mathbb{R}^{n-1} \times \mathbb{R}\right)\right\| \\
\quad+\sum_{j=1}^{m}\left\|\gamma_{j-1} u, H_{[\gamma]}^{\chi \cdot \mu_{j}}\left(\mathbb{R}^{n-1} \times \mathbb{R}\right)\right\| \\
\leq\left[\left\|u^{0}, H_{[\gamma]}^{r, \frac{r}{2 b}}\left(\mathbb{R}_{+}^{n} \times \mathbb{R}\right)\right\|+\left\|u^{1}, H_{[\gamma]}^{r, \frac{r}{2 b}}\left(\mathbb{R}_{+}^{n} \times \mathbb{R}\right)\right\|\right. \\
\left.\quad+\sum_{j=1}^{\kappa}\left\|\sigma_{j}, H_{[\gamma]}^{\chi \cdot \mu_{j+m}}\left(\mathbb{R}^{n-1} \times \mathbb{R}\right)\right\|+\sum_{j=1}^{m} \| \gamma_{j-1} u^{1}, H_{[\gamma]}^{\chi \cdot \mu_{j}}\left(\mathbb{R}^{n-1} \times \mathbb{R}\right)\right] .
\end{aligned}
$$

Using (5-16) we can estimate the first term on the right-hand side by a constant times $\left\|f, H_{[\gamma]}^{r-2 m, \frac{r-2 m}{2 b}}\left(\mathbb{R}_{+}^{n} \times \mathbb{R}\right)\right\|$. Using (5-18) we can estimate the second, third, and the fourth term by a constant times

$$
\begin{aligned}
\sum_{k=1}^{M}\left\|\tilde{g}_{k}, H_{[\gamma]}^{\chi \cdot \nu_{k}^{-1}}\left(\mathbb{R}^{n-1} \times \mathbb{R}\right)\right\| & \leq \sum_{k=1}^{M}\left\|g_{k}, H_{[\gamma]}^{\chi \cdot \nu_{k}^{-1}}\left(\mathbb{R}^{n-1} \times \mathbb{R}\right)\right\| \\
& +\sum_{k=1}^{M}\left\|\gamma_{0} B_{j}\left(D_{x}, D_{t}\right) u^{0}, H_{[\gamma]}^{\chi \cdot \nu_{j}^{-1}}\left(\mathbb{R}^{n-1} \times \mathbb{R}\right)\right\| .
\end{aligned}
$$

According to the compatibility condition the last sum can be estimated by a constant times $\left\|u^{0}, H_{[\gamma]}^{r, \frac{r}{2 b}}\left(\mathbb{R}_{+}^{n} \times \mathbb{R}\right)\right\|$ and consequently by a constant times

$$
\left\|f, H_{[\gamma]}^{r-2 m, \frac{r-2 m}{2 b}}\left(\mathbb{R}_{+}^{n} \times \mathbb{R}\right)\right\| .
$$

As a result we obtain the left inequality in (5-15).

The right inequality in (5-15) follows from the open mapping theorem or easily directly. In fact, since $f=A\left(D_{x}, D_{t}\right) u$, the corresponding term can be estimated by a constant times $\left\|u, H_{[\gamma]}^{r, \frac{r}{2 b}}\left(\mathbb{R}_{+}^{n} \times \mathbb{R}\right)\right\|$. According to our construction of the solution $\left(u, \sigma_{1}, \ldots, \sigma_{\kappa}\right)$, the relation (5-17) holds and the second sum in (5-15) can be estimated by

$$
\sum_{k=1}^{M}\left\|\tilde{g}_{k}, H_{[\gamma]}^{\chi \cdot \nu_{k}^{-1}}\left(\mathbb{R}^{n-1} \times \mathbb{R}\right)\right\|+\sum_{k=1}^{M}\left\|\gamma_{0} B_{j}\left(D_{x}, D_{t}\right) u^{0}, H_{[\gamma]+}^{\chi \cdot \nu_{j}^{-1}}\left(\mathbb{R}^{n-1} \times \mathbb{R}\right)\right\|
$$

According to (5-18) the first term can be estimated from above by

$$
\left\|u^{1}, H_{[\gamma]}^{r, \frac{r}{2 b}}\left(\mathbb{R}_{+}^{n} \times \mathbb{R}\right)\right\|+\sum_{j=1}^{\kappa}\left\|\sigma_{j}, H_{[\gamma]}^{\chi \cdot \mu_{j+m}}\left(\mathbb{R}^{n-1} \times \mathbb{R}\right)\right\| .
$$

Further, according to the compatibility condition

$$
\begin{aligned}
& \left\|u^{1}, H_{[\gamma]}^{r, \frac{r}{2 b}}\left(\mathbb{R}_{+}^{n} \times \mathbb{R}\right)\right\|+\sum_{k=1}^{M}\left\|\gamma_{0} B_{j}\left(D_{x}, D_{t}\right) u^{0}, H_{[\gamma]+}^{\chi \cdot \nu_{j}^{-1}}\left(\mathbb{R}^{n-1} \times \mathbb{R}\right)\right\| \\
& \quad \leq\left\|u^{1}, H_{[\gamma]}^{r, \frac{r}{2 b}}\left(\mathbb{R}_{+}^{n} \times \mathbb{R}\right)\right\|+C\left\|u^{0}, H_{[\gamma]}^{r, \frac{r}{2 b}}\left(\mathbb{R}_{+}^{n} \times \mathbb{R}\right)\right\| \\
& \leq\left\|u, H_{[\gamma]}^{r, \frac{r}{2 b}}\left(\mathbb{R}_{+}^{n} \times \mathbb{R}\right)\right\|+(C+1)\left\|u^{0}, H_{[\gamma]}^{r, \frac{r}{2 b}}\left(\mathbb{R}_{+}^{n} \times \mathbb{R}\right)\right\| .
\end{aligned}
$$


Now we can apply (5-16) to see that the last term can be estimated by a constant times $\left\|f, H_{[\gamma]}^{r-2 m, \frac{r-2 m}{2 b}}\left(\mathbb{R}_{+}^{n} \times \mathbb{R}\right)\right\|$. As it was mentioned above, this term is estimated by a constant times $\left\|u, H_{[\gamma]}^{r, \frac{r}{2 b}}\left(\mathbb{R}_{+}^{n} \times \mathbb{R}\right)\right\|$.

Remark 5.7. The two-sided estimate of Theorem 5.6 is based on the compatibility condition on $r, \mu_{i}, \nu_{i}$ and $\chi$. We will see in the next section that for the problems of crystallization such weight functions exist.

5.4. Construction of the weight-functions. Proof of Proposition 5.2. In this subsection we will apply the method of [12] to construct the weight-functions $\mu_{j}, \nu_{j} \in \mathcal{F}\left(\mathbb{R}^{n-1} \times \mathbb{C}_{-}\right)(j=1, \ldots, N)$ satisfying (5-3) and (5-4). Before we comment on the proof of Proposition 5.2, let us note some nonprincipal distinctions from $[12]$.

1) In [12] were considered matrices depending on real variables, say $\eta=\left(\xi^{\prime}, \sigma\right)$, and parameter, say $\gamma$. Here we have to consider matrices depending on a real variable $\xi^{\prime}$ and complex parameter $\tau=\sigma+i \gamma$.

2) In [12] all the constructions were made under additional assumption that the matrix is positively $N$-parameter elliptic or, in other words, all the functions $s_{i}(\rho)$ and $t_{i}(\rho)$ can be choosen nonnegative. In fact, this assumption is not necessary.

Indeed, we can choose $s_{i}(\rho), t_{i}(\rho)$ such that the functions $t_{i}(\rho)$ are nonnegative. Then we set

$$
L^{\prime}\left(\xi^{\prime}, \tau\right):=\left(\begin{array}{lll}
(i \tau+|\xi|)^{K} & & \\
& \ddots & \\
& & (i \tau+|\xi|)^{K}
\end{array}\right) L\left(\xi^{\prime}, \tau\right)
$$

for $K \in \mathbb{N}$ sufficiently large. We obtain

$$
\operatorname{ord}_{\rho} L_{j k}^{\prime}\left(\xi^{\prime}, \tau\right) \leq s_{j}^{\prime}(\rho)+t_{k}^{\prime}(\rho) \quad(j, k=1, \ldots, M)
$$

with $s_{j}^{\prime}(\rho):=s_{j}(\rho)+K \max \{1, \rho\}$ and $t_{k}^{\prime}(\rho):=t_{k}(\rho)$. By construction of the Lopatinskii matrix, $s_{j}(\rho)$ is not greater than a constant times $\rho$. Therefore, there exists a $K>0$ such that $s_{j}^{\prime}(\rho)>0(j=1, \ldots, M)$ for all $\rho>0$. For such $K$ the matrix $L\left(\xi^{\prime}, \tau\right)$ is positively $\mathrm{N}$-parameter-elliptic, and we can define the corresponding weight functions $\mu_{j}^{\prime}\left(\xi^{\prime}, \tau\right), \nu_{j}^{\prime}\left(\xi^{\prime}, \tau\right)$. Now we can take $\nu_{j}\left(\xi^{\prime}, \tau\right):=$ $(i \tau+|\xi|)^{-K} \nu_{j}\left(\xi^{\prime}, \tau\right)$ and $\mu_{j}^{\prime}\left(\xi^{\prime}, \tau\right):=\mu_{j}\left(\xi^{\prime}, \tau\right)$.

We will now comment on the proof of Proposition 5.2. The algorithm of [12] is based on a finite covering

$$
\left\{\left(\xi^{\prime}, \tau\right): \xi^{\prime} \in \mathbb{R}^{n-1}, \tau \in \mathbb{C}_{-}\right\}=\bigcup_{\ell=1}^{L} G_{\ell}(\varepsilon) \cup \bigcup_{\ell=1}^{L+1} \tilde{G}_{\ell}(\varepsilon)
$$

depending on a small parameter $\varepsilon$ and being defined by the Newton polygon $N(\operatorname{det} L)$. In each $G_{\ell}(\varepsilon)$ and $\tilde{G}_{\ell}(\varepsilon)$ we can explicitly define weight functions $\mu_{j}^{(\ell)}\left(\xi^{\prime}, \tau\right), \nu_{j}^{(\ell)}\left(\xi^{\prime}, \tau\right)$ and $\tilde{\mu}_{j}^{(\ell)}\left(\xi^{\prime}, \tau\right), \tilde{\nu}_{j}^{(\ell)}\left(\xi^{\prime}, \tau\right)$, respectively, such that $(5-3)-(5-4)$ are satisfied. Then using a partition of unity we "glue" the local weight functions to obtain global functions belonging to $\mathcal{F}$ and satisfying $(5-3)-(5-4)$ for all $\left(\xi^{\prime}, \tau\right)$.

Denote by $\left(p_{0}, q_{0}\right), \ldots,\left(p_{L+1}, q_{L+1}\right)$ the vertices of the Newton polygon $N(\operatorname{det} L)$, starting with $(0,0)$ and indexed in the clockwise direction. For $\ell=1, \ldots, L$ we choose $r_{\ell}>0$ such that the vector $\left(1, r_{\ell}\right)$ is an exterior normal to the edge from $\left(p_{\ell}, q_{\ell}\right)$ to $\left(p_{\ell+1}, q_{\ell+1}\right)$. By convexity we have $r_{1}>\cdots>r_{L}>0$. 
Following [5], Chapter 4, we define for sufficiently small $\varepsilon>0$ the domains

$$
G_{\ell}(\varepsilon):=\left\{\left(\xi^{\prime}, \tau\right) \in \mathbb{R}^{n-1} \times \mathbb{C}_{-}: \quad \varepsilon\left|\xi^{\prime}\right|^{r_{\ell}} \leq|\tau| \leq \varepsilon^{-1}\left|\xi^{\prime}\right|^{r_{\ell}}\right\} \quad(\ell=1, \ldots, L)
$$

corresponding to the edges of $N(\operatorname{det} L)$ and the domains

$$
\begin{aligned}
& \tilde{G}_{1}(\varepsilon):=\left\{\left(\left(\xi^{\prime} \tau\right)\right) \in \mathbb{R}^{n-1} \times \mathbb{C}_{-}: \quad \varepsilon^{-1}\left|\xi^{\prime}\right|^{r_{1}} \leq|\tau|\right\}, \\
& \left.\tilde{G}_{\ell}(\varepsilon):=\left\{\left(\xi^{\prime}, \tau\right)\right) \in \mathbb{R}^{n-1} \times \mathbb{C}_{-}: \quad \varepsilon^{-1}\left|\xi^{\prime}\right|^{r_{\ell}} \leq|\tau| \leq \varepsilon\left|\xi^{\prime}\right|^{r_{\ell-1}}\right\} \quad(\ell=2, \ldots, L), \\
& \tilde{G}_{L+1}(\varepsilon):=\left\{\left(\left(\xi^{\prime}, \tau\right)\right) \in \mathbb{R}^{n-1} \times \mathbb{C}_{-}: \quad|\tau| \leq \varepsilon\left|\xi^{\prime}\right|^{r_{L}}\right.
\end{aligned}
$$

corresponding to the vertices of $N(\operatorname{det} L)$.

We shall separately construct the weight functions in $G_{\ell}(\varepsilon)$ and in $\tilde{G}_{\ell}(\varepsilon)$.

For a given $\ell=1, \ldots, L$ we can find numbers, denote them as $s_{j}\left(r_{\ell}\right), t_{j}\left(r_{\ell}\right)(j=$ $1, \ldots, M)$ such that

$$
\begin{aligned}
\operatorname{ord}_{r_{\ell}}\left(L_{j k}\right) & \leq s_{j}\left(r_{\ell}\right)+t_{k}\left(r_{\ell}\right) \quad(j, k=1, \ldots, M), \\
\sum_{j=1}^{M}\left(s_{j}\left(r_{\ell}\right)+t_{j}\left(r_{\ell}\right)\right) & =\operatorname{ord}_{r_{\ell}}(\operatorname{det} L) .
\end{aligned}
$$

Now we can set

$$
\begin{aligned}
\nu_{j}^{(\ell)}\left(\xi^{\prime}, \tau\right) & :=\left(1+\left|\xi^{\prime}\right|+|\tau|^{1 / r_{\ell}}\right)^{s_{j}\left(r_{\ell}\right)}, \\
\mu_{j}^{(\ell)}\left(\xi^{\prime}, \tau\right) & :=\left(1+\left|\xi^{\prime}\right|+|\tau|^{1 / r_{\ell}}\right)^{t_{j}\left(r_{\ell}\right)}
\end{aligned}
$$

for $j=1, \ldots, M$.

The case of $\tilde{G}_{\ell}(\varepsilon)$ is more involved. First we consider the case $\ell=2, \ldots, L$. In this case we define

$$
\begin{aligned}
& \tilde{\nu}_{j}^{(\ell)}\left(\xi^{\prime}, \tau\right):=\left(1+\left|\xi^{\prime}\right|\right)^{\frac{r_{\ell-1} s_{j}\left(r_{\ell}\right)-r_{\ell} s_{j}\left(r_{\ell-1}\right)}{r_{\ell-1}-r_{\ell}}}(1+|\tau|)^{\frac{s_{j}\left(r_{\ell-1}\right)-s_{j}\left(r_{\ell}\right)}{r_{\ell-1}-r_{\ell}}}, \\
& \tilde{\mu}_{j}^{(\ell)}\left(\xi^{\prime}, \tau\right):=\left(1+\left|\xi^{\prime}\right|\right)^{\frac{r_{\ell-1} t_{j}\left(r_{\ell}\right)-r_{\ell} t_{j}\left(r_{\ell-1}\right)}{r_{\ell-1}-r_{\ell}}}(1+|\tau|)^{\frac{t_{j}\left(r_{\ell-1}\right)-t_{j}\left(r_{\ell}\right)}{r_{\ell-1}-r_{\ell}}}
\end{aligned}
$$

for $j=1, \ldots, M$. In the case $\ell=1$ we can take

$$
\tilde{\nu}_{j}^{(1)}\left(\xi^{\prime}, \tau\right):=(1+|\tau|)^{s_{j}\left(r_{1}\right) / r_{1}} \text { and } \tilde{\mu}_{j}^{(1)}\left(\xi^{\prime}, \tau\right):=(1+|\tau|)^{t_{j}\left(r_{1}\right) / r_{1}} .
$$

In the case $\ell=L+1$ we define

$$
\tilde{\nu}_{j}^{(L+1)}\left(\xi^{\prime}, \tau\right):=\left(1+\left|\xi^{\prime}\right|\right)^{s_{j}\left(r_{L+1}\right)} \text { and } \tilde{\mu}_{j}^{(L+1)}\left(\xi^{\prime}, \tau\right):=\left(1+\left|\xi^{\prime}\right|\right)^{t_{j}\left(r_{L+1}\right)} .
$$

It is shown in [5] that these weight functions satisfy (5-3)-(5-4) for sufficiently small $\gamma_{0}<0$.

5.5. Remarks about the existence of holomorphic weight functions. First of all note that in the construction presented in the previous subsection the local weights in the domains $G_{\ell}(\varepsilon)$ and $\tilde{G}_{\ell}(\varepsilon)$ can be chosen to be holomorphic in $\tau$. Indeed, for $\ell=1, \ldots, L$ the functions $(5-21)$ in $G_{\ell}(\varepsilon)$ are equivalent to

$$
\begin{aligned}
& \nu_{j}^{(\ell)}\left(\xi^{\prime}, \tau\right)=\left(1+\left|\xi^{\prime}\right|^{r_{\ell}}+i \tau\right)^{\frac{s_{j}\left(r_{\ell}\right)}{r_{\ell}}} \quad\left(j=1, \ldots, M,\left(\xi^{\prime}, \tau\right) \in G_{\ell}(\varepsilon)\right), \\
& \mu_{j}^{(\ell)}\left(\xi^{\prime}, \tau\right)=\left(1+\left|\xi^{\prime}\right|^{r_{\ell}}+i \tau\right)^{\frac{t_{j}\left(r_{\ell}\right)}{r_{\ell}}} \quad\left(j=1, \ldots, M,\left(\xi^{\prime}, \tau\right) \in G_{\ell}(\varepsilon)\right) .
\end{aligned}
$$

Analogously, the functions $(5-22)$ in $\tilde{G}_{\ell}(\varepsilon)$ are equivalent to

$$
\nu_{j}^{(\ell)}\left(\xi^{\prime}, \tau\right)=\left(1+\left|\xi^{\prime}\right|^{r_{\ell-1}}\right)^{a_{\ell}}(1+i \tau)^{b_{\ell}} \quad\left(j=1, \ldots, M,\left(\xi^{\prime}, \tau\right) \in \tilde{G}_{\ell}(\varepsilon)\right)
$$


where

$$
\begin{aligned}
a_{\ell} & :=\frac{r_{\ell-1} s_{j}\left(r_{\ell}\right)-r_{\ell} s_{j}\left(r_{\ell-1}\right)}{r_{\ell-1}\left(r_{\ell-1}-r_{\ell}\right)}, \\
b_{\ell} & :=\frac{s_{j}\left(r_{\ell-1}\right)-s_{j}\left(r_{\ell}\right)}{r_{\ell-1}-r_{\ell}} .
\end{aligned}
$$

Replacing $s_{j}\left(r_{\ell-1}\right)$ and $s_{j}\left(r_{\ell}\right)$ by $t_{j}\left(r_{\ell-1}\right)$ and $t_{j}\left(r_{\ell}\right)$, respectively, we define $\mu_{j}^{(\ell)}$.

The problem of gluing of these local weight functions in global holomorphic weight functions is open. We want to mention a simple sufficient condition which gives the answer in our examples. We suppose that the Newton polygon is a quadrangle.

Lemma 5.8. (i) Suppose that for some $j=1,2$ we have:

$$
\frac{s_{j}\left(r_{1}\right)}{r_{1}}=\frac{s_{j}\left(r_{2}\right)}{r_{2}}:=\kappa
$$

In that case we can take

$$
\nu_{j}\left(\xi^{\prime}, \tau\right)=\left(1+\left|\xi^{\prime}\right|^{r_{2}}+i \tau\right)^{\kappa} .
$$

(ii) Suppose that $s_{j}\left(r_{1}\right)=s_{j}\left(r_{2}\right):=s_{j}$. In that case we can take

$$
\nu_{j}\left(\xi^{\prime}, \tau\right)=\left(1+\left|\xi^{\prime}\right|^{r_{1}}+i \tau\right)^{\frac{s_{j}}{r_{1}}} .
$$

Proof. (i) First of all note that in our condition

$$
\begin{aligned}
a_{\ell} & =\frac{r_{\ell-1} s_{j}\left(r_{\ell}\right)-r_{\ell} s_{j}\left(r_{\ell-1}\right)}{r_{\ell-1}\left(r_{\ell-1}-r_{\ell}\right)}=0, \\
b_{\ell} & =\frac{s_{j}\left(r_{\ell-1}\right)-s_{j}\left(r_{\ell}\right)}{r_{\ell-1}-r_{\ell}}=\kappa .
\end{aligned}
$$

According to the construction in the preceding subsection, the function $\nu_{j}\left(\xi^{\prime}, \tau\right)$ is equivalent to

$$
|\tau|^{\kappa}, \quad\left(|\tau|+\left|\xi^{\prime}\right|^{r_{1}}\right)^{\kappa}, \quad|\tau|^{\kappa}, \quad\left(|\tau|+\left|\xi^{\prime}\right|^{r_{2}}\right)^{\kappa}, \quad\left|\xi^{\prime}\right|^{s_{j}\left(r_{2}\right)},
$$

in the domains $\tilde{G}_{1}, G_{1}, \tilde{G}_{2}, G_{2}, \tilde{G}_{3}$, respectively. Note that $|\tau| \geq C\left|\xi^{\prime}\right|^{r_{2}}$ in $\tilde{G}_{1} \cup$ $G_{1} \cup \tilde{G}_{2},\left|\xi^{\prime}\right|^{r_{2}} \geq C|\tau|$ in $G_{2} \cup \tilde{G}_{3}$, and $|\tau|+\left|\xi^{\prime}\right|^{r_{1}} \approx|\tau|+\left|\xi^{\prime}\right|^{r_{2}}$ in $G_{1}$. From this statement (i) follows.

(ii) In this case

$$
\begin{aligned}
a_{\ell} & =\frac{r_{\ell-1} s_{j}\left(r_{\ell}\right)-r_{\ell} s_{j}\left(r_{\ell-1}\right)}{r_{\ell-1}\left(r_{\ell-1}-r_{\ell}\right)}=s_{j}, \\
b_{\ell} & =\frac{s_{j}\left(r_{\ell-1}\right)-s_{j}\left(r_{\ell}\right)}{r_{\ell-1}-r_{\ell}}=0 .
\end{aligned}
$$

In the domains $\tilde{G}_{1}, G_{1}, \tilde{G}_{2}, G_{2}, \tilde{G}_{3}$ the function $\nu_{j}\left(\xi^{\prime}, \tau\right)$ is equivalent, respectively, to

$$
|\tau|^{\frac{s_{j}}{r_{1}}}, \quad\left(|\tau|+\left|\xi^{\prime}\right|^{r_{1}}\right)^{\frac{s_{j}}{r_{1}}}, \quad\left|\xi^{\prime}\right|^{s_{j}}, \quad\left(|\tau|+\left|\xi^{\prime}\right|^{r_{2}}\right)^{\frac{s_{j}}{r_{2}}}, \quad\left|\xi^{\prime}\right|^{s_{j}} .
$$

In the first function we can replace $|\tau|$ by $|\tau|+\left|\xi^{\prime}\right|^{r_{1}}$, in the last three functions we can replace $\left|\xi^{\prime}\right|$ by $\left(|\tau|+\left|\xi^{\prime}\right|^{r_{1}}\right)^{\frac{1}{r_{1}}}$. 


\section{ApplicAtion to THE PROBLEMS From CRYSTALLIZATION}

We will now apply the results of the preceding sections to the two problems from crystallization theory under consideration. We start with the Stefan problem. For this we fix $r \geq 2$ and define the weight function

$$
\lambda_{S}\left(\xi^{\prime}, \tau\right):=\left(1+\left|\xi^{\prime}\right|^{2}+i \tau\right)^{\frac{r}{2}-\frac{1}{4}}\left(1+\left|\xi^{\prime}\right|^{4}+i \tau\right)^{\frac{1}{2}} .
$$

Note that $\lambda_{S} \in \mathcal{F}_{\text {hol }}\left(\mathbb{R}^{n-1} \times \mathbb{C}_{-}\right)$. It can be seen easily that for all $r \geq 2$

$$
\lambda_{S}\left(\xi^{\prime}, \tau\right) \approx 1+|\tau|^{\frac{r}{2}+\frac{1}{4}}+\left|\xi^{\prime}\right|^{2}|\tau|^{\frac{r}{2}-\frac{1}{4}}+\left|\xi^{\prime}\right|^{r+\frac{3}{2}} .
$$

The case $r=2$ of the following theorem coincides with the estimate of [7] with $p=2$.

Theorem 6.1. Let $r \geq 2$. Then there exists a $\gamma_{0}<0$ such that for every $\gamma<$ $\gamma_{0}$ and for every $f \in H_{[\gamma]+}^{r-2, \frac{r}{2}-1}\left(\mathbb{R}_{+}^{n} \times \mathbb{R}\right), g \in H_{[\gamma]+}^{r-\frac{1}{2}, \frac{r}{2}-\frac{1}{4}}\left(\mathbb{R}^{n-1} \times \mathbb{R}\right)$ and $h \in$ $H_{[\gamma]+}^{r-\frac{3}{2}, \frac{r}{2}-\frac{3}{4}}\left(\mathbb{R}^{n-1} \times \mathbb{R}\right)$ the boundary value problem (1-4) has a unique solution

$$
(u, \sigma) \in H_{[\gamma]+}^{r, \frac{r}{2}}\left(\mathbb{R}_{+}^{n} \times \mathbb{R}\right) \times H_{[\gamma]_{+}}^{\lambda_{S}}\left(\mathbb{R}^{n-1} \times \mathbb{R}\right) .
$$

Moreover, the two-sided a priori estimate

$$
\begin{aligned}
& \left\|u, H_{[\gamma]+}^{r, \frac{r}{2}}\left(\mathbb{R}_{+}^{n} \times \mathbb{R}\right)\right\|+\left\|\sigma, H_{[\gamma]_{+}}^{\lambda_{S}}\left(\mathbb{R}^{n-1} \times \mathbb{R}\right)\right\| \\
& \approx\left\|f, H_{[\gamma]+}^{r-2, \frac{r}{2}-1}\left(\mathbb{R}_{+}^{n} \times \mathbb{R}\right)\right\|+\left\|g, H_{[\gamma]+}^{r-\frac{1}{2}, \frac{r}{2}-\frac{1}{4}}\left(\mathbb{R}^{n-1} \times \mathbb{R}\right)\right\| \\
& \quad+\left\|h, H_{[\gamma]+}^{r-\frac{3}{2}, \frac{r}{2}-\frac{3}{4}}\left(\mathbb{R}^{n-1} \times \mathbb{R}\right)\right\|
\end{aligned}
$$

holds.

Proof. In Subsection 3.5 we defined $s_{1}(\rho):=t_{1}(\rho):=0, s_{2}(\rho):=\max \left\{1, \frac{\rho}{2}\right\}$ and $t_{2}(\rho):=\max \left\{2, \frac{\rho}{2}\right\}$. As the Newton polygon has the vertices $(0,0),(0,1),\left(2, \frac{1}{2}\right)$ and $(3,0)$ (see Figure 1$)$, the exterior normals are $\left(1, r_{1}\right)$ and $\left(1, r_{2}\right)$ with $r_{1}=4, r_{2}=2$ (see (4-7)). Because of

$$
\frac{s_{2}(4)}{4}=\frac{s_{2}(2)}{2}=\frac{1}{2}
$$

we can apply Lemma 5.8 (i) and obtain

$$
\nu_{2}\left(\xi^{\prime}, \tau\right)=\left(1+\left|\xi^{\prime}\right|^{2}+i \tau\right)^{\frac{1}{2}} .
$$

In the same way we have $t_{2}(4)=t_{2}(2)=2$, and an application of Lemma 5.8 (ii) gives $\mu_{2}\left(\xi^{\prime}, \tau\right)=\left(1+\left|\xi^{\prime}\right|^{4}+i \tau\right)^{\frac{1}{2}}$. Setting $\mu_{1}\left(\xi^{\prime}, \tau\right)=\nu_{1}\left(\xi^{\prime}, \tau\right)=1$, we obtain the weight functions $\mu_{1,2}, \nu_{1,2} \in \mathcal{F}_{\text {hol }}\left(\mathbb{R}^{n-1} \times \mathbb{C}_{-}\right)$corresponding to the Stefan problem.

Now it is easily seen that for the weight function

$$
\chi\left(\xi^{\prime}, \tau\right):=\left(1+\left|\xi^{\prime}\right|^{2}+i \tau\right)^{\frac{r}{2}-\frac{1}{4}}
$$

the compatibility condition holds (in fact, up to equivalence it is the only possible choice). So all assumptions of Theorem 5.4 are satisfied, and an application of this 
theorem gives the desired result. Note that

$$
\begin{aligned}
\left|\left(\chi \cdot \mu_{1}\right)\left(\xi^{\prime}, \tau\right)\right| & =\left|\chi\left(\xi^{\prime}, \tau\right)\right| \approx 1+\left|\xi^{\prime}\right|^{r-\frac{1}{2}}+|\tau|^{\frac{r}{2}-\frac{1}{4}}, \\
\left|\left(\chi \cdot \mu_{2}\right)\left(\xi^{\prime}, \tau\right)\right| & =\left|\lambda_{S}\left(\xi^{\prime}, \tau\right)\right| \approx 1+|\tau|^{\frac{r}{2}+\frac{1}{4}}+\left|\xi^{\prime}\right|^{2}|\tau|^{\frac{r}{2}-\frac{1}{4}}+\left|\xi^{\prime}\right|^{r+\frac{3}{2}}, \\
\left|\left(\chi \cdot \nu_{1}^{-1}\right)\left(\xi^{\prime}, \tau\right)\right| & =\left|\chi\left(\xi^{\prime}, \tau\right)\right| \approx 1+\left|\xi^{\prime}\right|^{r-\frac{1}{2}}+|\tau|^{\frac{r}{2}-\frac{1}{4}} \\
\left|\left(\chi \cdot \nu_{2}^{-1}\right)\left(\xi^{\prime}, \tau\right)\right| & \approx 1+\left|\xi^{\prime}\right|^{r-\frac{3}{2}}+|\tau|^{\frac{r}{2}-\frac{3}{4}} .
\end{aligned}
$$

Therefore, the norms of Theorem 5.4 coincide with the norms of Theorem 6.1. The term $\left\|\gamma_{0} u, \ldots\right\|$ in Theorem 5.4 can be omitted here as

$$
H_{[\gamma]+}^{\chi \cdot \mu_{1}}\left(\mathbb{R}^{n-1} \times \mathbb{R}\right)=H_{[\gamma]+}^{r-\frac{1}{2}, \frac{r}{2}-\frac{1}{4}}\left(\mathbb{R}^{n-1} \times \mathbb{R}\right)
$$

in this case.

For the Cahn-Hilliard equation with dynamic boundary conditions (1-5), we fix $r \geq 4$ and define

$$
\lambda_{C}\left(\xi^{\prime}, \tau\right):=\left(1+\left|\xi^{\prime}\right|^{2}+i \tau\right)\left(1+\left|\xi^{\prime}\right|^{4}+i \tau\right)^{\frac{r}{4}-\frac{3}{8}} .
$$

Elementary calculations show that

$$
\left|\lambda_{C}\left(\xi^{\prime}, \tau\right)\right| \approx 1+\left|\xi^{\prime}\right|^{r+\frac{1}{2}}+|\tau|^{\frac{r}{4}+\frac{5}{8}}+\left|\xi^{\prime}\right|^{r-\frac{3}{2}}|\tau| .
$$

Theorem 6.2. Let $r \geq 4$. Then there exists a $\gamma_{0}<0$ such that for every $\gamma<$ $\gamma_{0}$ and for every $f \in H_{[\gamma]+}^{r-4, \frac{r}{4}-1}\left(\mathbb{R}_{+}^{n} \times \mathbb{R}\right), g \in H_{[\gamma]+}^{r-\frac{7}{2}, \frac{r}{4}-\frac{7}{8}}\left(\mathbb{R}^{n-1} \times \mathbb{R}\right)$ and $h \in$ $H_{[\gamma]+}^{r-\frac{3}{2}, \frac{r}{4}-\frac{3}{8}}\left(\mathbb{R}^{n-1} \times \mathbb{R}\right)$ the boundary value problem (1-5) has a unique solution

$$
u \in H_{[\gamma]+}^{r, \frac{r}{4}}\left(\mathbb{R}_{+}^{n} \times \mathbb{R}\right) .
$$

Additionally, the trace $\gamma_{0} u$ belongs to $H_{[\gamma]+}^{\lambda_{C}}\left(\mathbb{R}^{n-1} \times \mathbb{R}\right)$, and the two-sided a priori estimate

$$
\begin{aligned}
\left\|u, H_{[\gamma]+}^{r, \frac{r}{4}}\left(\mathbb{R}_{+}^{n} \times \mathbb{R}\right)\right\|+ & \left\|\gamma_{0} u, H_{[\gamma]+}^{\lambda_{C}}\left(\mathbb{R}^{n-1} \times \mathbb{R}\right)\right\| \\
\approx & \left\|f, H_{[\gamma]+}^{r-4, \frac{r}{4}-1}\left(\mathbb{R}_{+}^{n} \times \mathbb{R}\right)\right\|+\left\|g, H_{[\gamma]+}^{r-\frac{7}{2}, \frac{r}{4}-\frac{7}{8}}\left(\mathbb{R}^{n-1} \times \mathbb{R}\right)\right\| \\
& +\left\|h, H_{[\gamma]+}^{r-\frac{3}{2}, \frac{r}{4}-\frac{3}{8}}\left(\mathbb{R}^{n-1} \times \mathbb{R}\right)\right\|
\end{aligned}
$$

holds.

Proof. The proof follows the same lines as the proof of Theorem 6.1. We have seen in Subsection 3.6 that the mixed-order structure of the Lopatinskii matrix for (1-2) is given by $s_{1}(\rho)=\max \left\{2, \frac{\rho}{2}\right\}, t_{1}(\rho)=\max \{2, \rho\}, s_{2}(\rho)=t_{2}(\rho)=0$. From (4-7) we see $r_{1}=4$ and $r_{2}=2$.

As we have

$$
\frac{t_{1}(4)}{4}=\frac{t_{1}(2)}{2}=1
$$

we can apply Lemma 5.8 (i) and get

$$
\mu_{1}\left(\tau, \xi^{\prime}\right)=1+\left|\xi^{\prime}\right|^{2}+i \tau
$$

In the same way

$$
s_{1}(4)=s_{1}(2)=2 \text { and } \frac{s_{1}}{r_{1}}=\frac{1}{2}
$$


and according to Lemma 5.8 (ii) we can take

$$
\nu_{1}\left(\tau, \xi^{\prime}\right)=\left(1+\left|\xi^{\prime}\right|^{4}+i \tau\right)^{\frac{1}{2}} .
$$

Obviously, $\nu_{2}\left(\tau, \xi^{\prime}\right)=\mu_{2}\left(\tau, \xi^{\prime}\right)=1$.

It is easily seen that for the weight function

$$
\chi\left(\xi^{\prime}, \tau\right):=\left(1+\left|\xi^{\prime}\right|^{4}+i \tau\right)^{\frac{r}{4}-\frac{3}{8}}
$$

the compatibility condition is satisfied. We can apply Theorem 5.4 and obtain unique solvability and the two-sided a priori estimate. Here the norms are given by

$$
\begin{aligned}
\left|\left(\chi \cdot \mu_{1}\right)\left(\xi^{\prime}, \tau\right)\right| & =\left|\lambda_{C}\left(\xi^{\prime}, \tau\right)\right| \approx 1+\left|\xi^{\prime}\right|^{r+\frac{1}{2}}+|\tau|^{\frac{r}{4}+\frac{5}{8}}+\left|\xi^{\prime}\right|^{r-\frac{3}{2}}|\tau|, \\
\left|\left(\chi \cdot \mu_{2}\right)\left(\xi^{\prime}, \tau\right)\right| & =\left|\chi\left(\xi^{\prime}, \tau\right)\right| \approx 1+\left|\xi^{\prime}\right|^{r-\frac{3}{2}}+|\tau|^{\frac{r}{4}-\frac{3}{8}} \\
\left|\left(\chi \cdot \nu_{1}^{-1}\right)\left(\xi^{\prime}, \tau\right)\right| & \approx 1+\left|\xi^{\prime}\right|^{r-\frac{7}{2}}+|\tau|^{\frac{r}{4}-\frac{7}{8}} \\
\left|\left(\chi \cdot \nu_{2}^{-1}\right)\left(\xi^{\prime}, \tau\right)\right| & =\left|\chi\left(\xi^{\prime}, \tau\right)\right| \approx 1+\left|\xi^{\prime}\right|^{r-\frac{3}{2}}+|\tau|^{\frac{r}{4}-\frac{3}{8}}
\end{aligned}
$$

The term for $\gamma_{1} u$ in Theorem 5.4 can be omitted but the estimate for the norm of $\gamma_{0} u$ gives an additional smoothness and a corresponding term in the a priori estimate.

For the case $r=4$ the estimate of Theorem 6.2 can be found in [8] where also the $L_{p}$-spaces with $p \neq 2$ are considered.

\section{REFERENCES}

[1] S. Agmon, On the eigenfunctions and on the eigenvalues of general elliptic boundary value problems, Comm. Pure Appl. Math., 15 (1962), 119-147.

[2] Agmon S., Douglis A., and Nirenberg L. : Estimates near the boundary for solutions of elliptic partial differential equations satisfying general boundary conditions I. , Comm. Pure Appl. Math. 12, (1959), 623-727.

[3] Agranovich, M. S., Vishik, M. I.: Elliptic problems with parameter and parabolic problems of general form (Russian). Uspekhi Mat. Nauk, 19 (1964), No. 3, 53-161. English transl. in Russian Math. Surv. 19 (1964), No. 3, 53-157.

[4] Eidel'man, S.D.: Parabolic systems. Wolters-Noordhoff Publishing Groningen, 1969.

[5] Gindikin, S. G., Volevich, L. R.: The Method of Newton's Polyhedron in the Theory of Partial Differential Equations. Math. Appl. (Soviet Ser.) 86, Kluwer Academic, Dordrecht, 1992.

[6] Gindikin, S. G., Volevich, L. R.: Mixed Problem for Partial Differential Operators with Quasihomogeneous Principal Part, Translations of Mathematical Monographs, vol. 147, 1996, Amer. Math. Soc., Providence, Rhode Island, 233 p.

[7] Escher, J., Prüss, J., Simonett, G.: Analytic solutions for a Stefan problem with GibbsThomson correction. J. Reine Angew. Math. 563, 1-52 (2003).

[8] Prüss, J., Racke, R., Zheng, S.: Maximal regularity and asymptotic behavior of solutions for the Cahn-Hilliard equation with dnnamic boundary conditions. Preprint Konstanzer Schriften in Mathematik und Informatik 189 (2003), 21 pp.

[9] Roitberg, Y.: Boundary value problems in the spaces of distributions. Mathematics and its Applications, 498. Kluwer Academic Publishers, Dordrecht, 1999.

[10] Solonnikov, V. A.: On boundary value problems for linear parabolic systems of differential equations of general form (Russian). Trudy Mat. Inst. Steklov. 83, Leningrad, 1965.

[11] Volevich, L. R.: One Problem of Linear Programming Arising in Partial Differential Equations (Russian). Uspekhi Mat. Nauk 18 (1963), No. 3, 155-162.

[12] Volevich, L. R.: Newton polygon and general parameter-elliptic (parabolic) systems. Russian J. Math. Phys. 8 (2001), 375-400.

[13] Volevich, L. R., Paneah, B. P.: Some spaces of generalized functions and embedding theorems (Russian). Uspehi Mat. Nauk 20 (1965), No. 1 (121), 3-74. English transl. in Russian Math. Surv. 20 1964, No. 1, 1-73. 